\title{
A Numerical PDE Approach For Pricing Callable Bonds
}

\author{
Y. d'Halluin; P.A. Forsyth† K.R. Vetzalł and G. Labahn ${ }^{\S}$ \\ University of Waterloo \\ Waterloo, Ontario \\ Canada N2L 3G1
}

February 2, 2001

\begin{abstract}
Many debt issues contain an embedded call option that allows the issuer to redeem the bond at specified dates for a specified price. The issuer is typically required to provide advance notice of a decision to exercise this call option. The valuation of these contracts is an interesting numerical exercise because discontinuities may arise in the bond value or its derivative at call and/or notice dates. Recently, it has been suggested that finite difference methods cannot be used to price callable bonds requiring notice (Büttler, 1995; Büttler and Waldvogel, 1996). Poor accuracy was attributed to discontinuities and difficulties in handling boundary conditions. As an alternative, a semi-analytical method using Green's functions for valuing callable bonds with notice was proposed (Büttler and Waldvogel, 1996). Unfortunately, the Green's function method is limited to special cases. Consequently, it is desirable to develop a more general approach. We provide this by using more advanced techniques such as flux limiters to obtain an accurate numerical partial differential equation method. Finally, in a typical pricing model (Cox et al., 1985) an inappropriate financial condition is required in order to properly specify boundary conditions for the associated PDE. We show that a small perturbation of such a model is free from such artificial conditions.
\end{abstract}

Keywords: Callable bond, numerical PDE, discontinuity, Green's function

\footnotetext{
${ }^{*}$ Department of Computer Science, ydhallui@elora.uwaterloo.ca

${ }^{\dagger}$ Department of Computer Science, paforsyt@elora.uwaterloo.ca

${ }^{\ddagger}$ Centre for Advanced Studies in Finance, kvetzal@watarts.uwaterloo.ca

${ }^{\S}$ Department of Computer Science, glabahn@daisy.uwaterloo.ca

"Acknowledgement: This work was supported by the Natural Sciences and Engineering Research Council of Canada, the Royal Bank of Canada, and the Social Sciences and Humanities Research Council of Canada.
} 


\section{Introduction}

Interest rate derivative securities comprise the largest segment of the over-the-counter derivatives market, having a total notional amount outstanding in excess of $\$ 60$ trillion at the end of 1999 (Bank for International Settlements, 2000). A large number of different models have been proposed to value and hedge these securities. It is beyond the scope of this paper to review this literature. Interested readers are referred to sources such as Hughston (1996) or Hull (2000) for further information and references. There are, however, two general approaches. The first begins with a model for the evolution of the instantaneous risk free interest rate $r$ and proceeds via hedging arguments to obtain a partial differential equation (PDE) which can be solved subject to appropriate boundary conditions to value interest rate derivative securities. Two well-known examples of this approach are contained in the papers by Vasicek (1977) and Cox et al. (hereafter CIR) (1985). While this approach is fairly straightforward, it suffers from the drawback that it is not automatically consistent with observed market prices for bonds. One way to circumvent this is to make some of the parameters in the model time-dependent, as suggested by Hull and White (1990) among others. The second approach, originated by Heath et al. (1992), involves modelling the movements of the entire yield curve from the start. Since this takes the current term structure as an input to the model, there is no need for time-dependent parameters. Unfortunately, this approach produces models which are in general path-dependent and very difficult to implement. The only general purpose technique which can be used for these types of models is Monte Carlo simulation. This suffers from the drawback of being relatively slow. Moreover, Monte Carlo methods are typically problematic to apply for American-style securities. Consequently, some authors have devoted attention to special cases which are either Markovian or which have the property that the path-dependency can be captured by a small number of additional state variables (e.g. Ritchken and Sankarasubramanian, 1995; Bhar et al., 2000).

Our focus in this paper is on callable bonds. A callable bond is a simple coupon-bearing bond with an embedded option that allows the issuer to call the bond back at specified future dates for a specified price. We concentrate on the first approach described above, where we have a PDE representation of the value of the contract. By solving the PDE backward in time, optimal decision making can be modelled in a straightforward manner. It is worth noting, however, that our methods could be applied to special cases of the second approach such as Bhar et al. (2000). In addition, although we concentrate on default risk free contracts, under certain assumptions callable corporate debt issues subject to default risk could be handled by replacing the risk free rate $r$ with a risk-adjusted version, as in Duffie and Singleton (1999).

In practice, most callable bonds require that the issuer provide advance notice of a decision to exercise the embedded call. A typical notice period would be in the range of one to four months. Though often ignored in the literature (e.g. Brennan and Schwartz, 1977), this prior notice feature has several interesting implications:

1. Discontinuities can arise in either the solution profile for a callable bond or its derivative with respect to $r$ at call and/or notice dates. As will be discussed in greater detail below, this can produce problems such as spurious oscillations with numerical valuation schemes if not handled appropriately. Indeed, Büttler (1995) suggested that it was not possible to accurately value this type of contract using standard numerical PDE methods. We show that this conclusion does not hold if an alternative discretization scheme is used. It also is worth pointing out that our methods are applicable to any case where advance notice of exercise must be provided. For example, putable bond contracts also often have this feature (Crabbe and Nikoulis, 1997). 
2. The standard description of the optimal call policy for the issuer is to call the bond as soon as its value reaches the call price (Brennan and Schwartz, 1977, p. 75). As noted by Bliss and Ronn (1998), this is no longer correct when advance notice must be provided. ${ }^{1}$ As an alternative, Bliss and Ronn introduce a "threshold volatility" level and compare it to prevailing implied volatilities in order to determine whether calling is optimal when advance notification is required. This approach requires iterative numerical searching to determine the inputs for this volatility comparison. By contrast, the approach proposed in this paper and described in further detail below avoids this searching and thus appears (to us at least) to be simpler.

It is possible to derive an analytic expression for the Green's function of the contingent claim PDE for certain specific interest rate models. Büttler and Waldvogel (1996) follow this line and develop a semi-analytic method for valuing callable bonds with notice. In addition to only being applicable for specific models, this approach suffers from the drawback that the parameters of the models are assumed to be constant. This precludes the use of time-dependent parameters to calibrate the model to the current term structure. Our fully numerical approach is not limited in this way. It is straightforward to extend the methods described in this paper to cases where the Green's function cannot be determined analytically, as well as to cases with time-dependent parameters, or to models with more than one factor such as versions of the models proposed by CIR (1985) or Duffie and Kan (1996) (among many others) which have two or three state variables. However, in this paper we concentrate on single factor models with constant parameters which allow for solutions using the Green's function method of Büttler and Waldvogel (1996). This permits comparison with our discretized PDE technique.

Finally, when considering numerical PDE methods, issues arise with regard to the discretization of the boundary conditions. In particular, when we study single factor interest rate models with positive interest rate domains such as that of CIR (1985), we need to analyze carefully the boundary condition at $r=0$ in order to avoid unneccessary restrictions on our models. We show that a slightly perturbed CIR model avoids such restrictions and allows for a simple discretization at the boundary.

The outline of this paper is as follows. Section 2 describes the modelling framework and introduces some definitions. Section 3 discusses two methods to solve the PDE model. In Section 4, we study the boundary condition at $r=0$ for a single factor interest rate model. Section 5 contains numerical results, and conclusions are provided in Section 6.

\section{Background}

To fix ideas and notation, consider a single factor model. This factor is the instantaneous risk free interest rate $r$ which is assumed to follow a stochastic process of the form

$$
d r=f(r, t) d t+\sigma(r, t) r^{\beta} d z,
$$

where $f(r, t)$ is the instantaneous drift, $\sigma(r, t) r^{\beta}$ is the instantaneous volatility, and $d z$ is the increment of a Wiener process. Note that equation (1) contains the well-known models of Vasicek (1977) and CIR (1985) as special cases. In particular, if $f(r, t)$ is specified to be mean-reverting

\footnotetext{
${ }^{1}$ There are other factors which can also cause the standard description of the optimal call policy to be incorrect. Examples include market imperfections such as transactions costs (Mauer, 1993) or, in the case of corporate bonds, capital structure changes (Longstaff and Tuckman, 1994). We ignore these alternative factors below, concentrating exclusively on the effect of the advance notice provision and assuming that the issuer seeks to minimize the value of the contract.
} 
and independent of time $t$, and $\sigma(r, t)$ is a constant, then the restriction $\beta=0$ produces the Vasicek model and $\beta=1 / 2$ corresponds to the CIR model. Based on standard hedging arguments, a PDE for the value $P(r, T)$ of an interest rate contingent claim is

$$
P_{\tau}=\frac{1}{2} \sigma(r, \tau)^{2} r^{2 \beta} P_{r r}+\left(f(r, \tau)+\sigma(r, \tau) q(r, \tau) r^{\beta}\right) P_{r}-r P,
$$

where $\tau=T-t$ represents time evolving backward from the expiration date of the claim $T$ to the current time $t$ and $q(r, \tau)$ is the market price of interest rate risk.

Various different types of claims can be valued by specifying suitable boundary and initial conditions and solving the backward (in time) equation (2). Before considering some examples, we remark that in equation (2) the term

$$
\frac{1}{2} \sigma(r, \tau)^{2} r^{2 \beta} P_{r r}
$$

is a diffusion term, while

$$
\left(f(r, \tau)+\sigma(r, \tau) q(r, \tau) r^{\beta}\right) P_{r}
$$

is a first-order hyperbolic convective term. This latter term propagates information with a velocity of $-\left(f(r, t)+\sigma(r, t) q(r, \tau) r^{\beta}\right)$. If it is large relative to the diffusion term, equation (2) is said to be convection-dominant. It can then become difficult to solve accurately using standard numerical methods.

The simplest type of claim which can be valued using equation (2) is a $T$ year zero coupon bond paying some fixed principal amount at maturity. This contract would involve the initial condition

$$
P(r, \tau=0)=\text { Principal. }
$$

The boundary conditions will depend on the particular interest rate model considered. These will be discussed in further detail below. A slightly less trivial example is a coupon-bearing bond, paying $C$ (in dollars) at times $t_{c_{i}}$ for $i=1, \ldots, M$ where $M$ is the number of coupon payments prior to the maturity date $T$, plus a final coupon payment at $T$. The initial condition becomes

$$
P(r, \tau=0)=\text { Principal }+C .
$$

We then solve equation (2), enforcing the constraint

$$
P\left(r, \tau_{c_{i}}^{+}\right)=P\left(r, \tau_{c_{i}}^{-}\right)+C
$$

at each coupon payment date, where $\tau_{c_{i}}^{+}\left(\tau_{c_{i}}^{-}\right)$is the value of the bond an instant after (before) the coupon payment.

We now turn to our main topic of callable bonds. Following Büttler and Waldvogel (1996), we distinguish three types of these contracts:

1. European callable bond: the issuer has the right to call the bond at only one date (typically the last coupon date before maturity).

2. American callable bond: the issuer may call the bond at any time.

3. Semi-American callable bond: the issuer has the right to call the bond at one of a specified set of dates (usually coinciding with coupon dates). This type of contract is also known as a Bermudan callable bond. 
Note that in the American/Bermudan cases, there is usually a "lock-out" period, defined as the length of time from issuance until the first possible call date. A representative lock-out period might be five years, but the range is from as short as a month to more than ten years. The most common type is the semi-American contract, and we will concentrate primarily on this case in the following.

For simplicity, however, we will first discuss the European case with a single possible call date, this being the last coupon date prior to maturity. We denote this call/coupon date by $t_{c_{M}}$ going forward in time and define $\tau_{c_{M}}=T-t_{c_{M}}$. Similarly, the notice date is $t_{n_{M}}$, and $\tau_{n_{M}}=T-t_{n_{M}}$. Going backward in time, we also define $\tau_{n_{M}}^{+}\left(\tau_{n_{M}}^{-}\right)$as the time immediately after (before) the notice date (see Figure 1). The value of this European callable bond $K(r, T)$ can be calculated as follows. Let $X\left(t_{c_{M}}\right)$ denote the call price. As noted above, we will assume that it is optimal for the issuer to minimize the value of the contract. Thus, the issuer will exercise the option if the value of the callable bond exceeds the prevailing present value of the call price plus the coupon payment. The interest rate at the notice date $t_{n_{M}}$ where the issuer is indifferent between exercising the option or not doing so is called the "break-even" interest rate, $r_{b}$. This rate may be found by setting the value of the callable bond immediately before the notice date (going backward in time) equal to the discounted value of the call price

$$
\left[X\left(t_{c_{M}}\right)+C\right] P\left(r_{b}, \tau_{n_{M}}-\tau_{c_{M}}\right)-K\left(r_{b}, \tau_{n_{M}}^{-}\right)=0,
$$

where $K\left(r_{b}, \tau_{n_{M}}^{-}\right)$denotes the value of the callable bond an instant before the notice date and where $P(r, \tau)$ is the discrete solution of equation (2) with initial condition $P(r, 0)=1$ (thus, $P\left(r, \tau_{n_{M}}-\tau_{c_{M}}\right)$ is the value at $\tau_{n_{M}}$ of a zero coupon bond maturing at $\tau_{c_{M}}$ with face value of unity).

Once the break-even interest rate is found we need to update the price of the callable bond. For the typical contractual specification, this should happen at the notice date. This will be referred to as "Method 1" in this paper. Hence, the value of the callable bond an instant after the notice date (solving backward in time) is

$$
K^{\text {method }_{1}}\left(r, \tau_{n_{M}}^{+}\right)= \begin{cases}{[X+C] P\left(r, \tau_{n_{M}}-\tau_{c_{M}}\right)} & \text { if } r \leq r_{b}, \\ K\left(r, \tau_{n_{M}}^{-}\right) & \text {otherwise }\end{cases}
$$

Then, we solve equation (2) with $K^{\operatorname{method}_{1}}\left(r, \tau_{n_{M}}^{+}\right)$as the initial condition at $t=t_{n_{M}}$ back to the present time $t=t_{0}$, adding coupon payments along the way as described in equation (3). This type of approach appears to have been followed by Büttler and Waldvogel (1996). However, it is puzzling to note that Büttler (1995, p. 379) stated that updating the solution creates a discontinuity. This is because a discontinuity cannot occur in this situation since the solution is updated at $\tau=\tau_{n_{M}}$ by taking $K\left(r, \tau_{n_{M}}^{+}\right)=\min \left([X+C] P\left(r, \tau_{n_{M}}-\tau_{c_{M}}\right), K\left(r, \tau_{n_{M}}^{-}\right)\right)$. A representative example illustrating this for a particular case of the Vasicek (1977) model is provided in Figure 2(a). As the figure reveals, there is no discontinuity in the solution profile, although there is in the derivative of the solution. This could cause difficulties in obtaining accurate numerical estimates of hedging parameters unless certain precautions are taken.

In order to provide a more stringent test for our numerical methods, it is instructive to consider a slight variation of the typical callable bond contract. For the standard contract, recall that the issuer announces on the notice date whether or not the bond will be called at the next call date. The variation which we will study provides the issuer with some additional optionality, and thus will lead to higher valuations for the embedded call feature. On the notice date, the issuer announces that the bond will be called at the next call date if the prevailing value of $r$ at the call date is less 
than or equal to some level $\hat{r}$. This will create a discontinuity in the solution profile at the call date. This will be referred to as "Method 2". From the issuer's perspective, the choice of $\hat{r}$ presents an interesting optimization problem. To solve this would require using higher dimensional methods, and is beyond the scope of this paper. As our main purpose here is to study the numerical effects of discontinuities, we shall simply assume that $\hat{r}=r_{b}$, i.e. the issuer selects the same rate as the break-even rate used in Method 1.

Figures 2(b) and 2(c) illustrate the discontinuity that arises using Method 2. For this contract, the price of the callable bond an instant after the call date (going backward in time) is given by

$$
K^{\text {method }_{2}}\left(r, \tau_{c_{M}}^{+}\right)= \begin{cases}{[X+C]} & \text { if } r \leq r_{b}, \\ K\left(r, \tau_{c_{M}}^{-}\right) & \text {otherwise. }\end{cases}
$$

We then solve equation (2) with $K^{\text {method }_{2}}\left(r, \tau_{c_{M}}^{+}\right)$as the initial condition at $t_{c_{M}}$ back to the present time $t_{0}$, incorporating coupon payments as required.

We can now generalize these approaches to the more complicated and common case of the semi-American callable bond. Suppose we have a $T$ year bond with $M$ coupon payments prior to $T$ and $N<M$ call dates that coincide with the last $N$ coupon payment dates before $T$. We denote the coupon dates by $t_{c_{i}}, i=1, \ldots, M$ and the notice dates by $t_{n_{j}}, j=1, \ldots, N$. Similarly, $\tau_{n_{j}}=T-t_{n_{j}}$ represents the time from the maturity date to notice date $j$, while $\tau_{c_{i}}=T-t_{c_{i}}$ represents the time from the maturity date to coupon date $i$. As for the European callable bond, we work backward in time. An outline of the algorithm is as follows:

1. Solve equation (2) from $T$ to $t_{c_{M}}$ with initial condition

$$
K(r, \tau=0)=\text { Principal }+C .
$$

The solution is denoted by $K\left(r, \tau_{c_{M}}^{-}\right)$.

2. Add the coupon payment to the solution

$$
K\left(r, \tau_{c_{M}}^{+}\right)=K\left(r, \tau_{c_{M}}^{+}\right)+C
$$

3. Solve for the present value of the call price

$$
\left[X\left(t_{c_{M}}\right)+C\right] P\left(r, \tau_{n_{M}}-\tau_{c_{M}}\right)
$$

from the call date $\tau_{c_{M}}$ to the notice date $\tau_{n_{M}}^{-}$. Also solve (2) with $K\left(r, \tau_{c_{M}}^{+}\right)$as initial condition for the same period, and denote the solution an instant before the notice date by $K\left(r, \tau_{n_{M}}^{-}\right)$. Next compute the break-even interest rate using

$$
\left[X\left(t_{c_{M}}\right)+C\right] P\left(r_{b}, \tau_{n_{M}}-\tau_{c_{M}}\right)-K\left(r_{b}, \tau_{n_{M}}^{-}\right)=0 .
$$

Apply the solution updating method appropriate for either type of contract specification (i.e. Method 1 or Method 2) and solve equation (2) to the next coupon payment.

4. Repeat steps 2-3 for the remaining call dates.

5. Solve back to the present $t_{0}$, adding coupon payments at the remaining coupon dates as in equation (3). 


\section{Solving the One Factor Interest Rate Model}

In this section, we discuss two methods to solve the PDE (2) for semi-American callable bonds with notice. We begin by reviewing the Green's function approach suggested by Büttler and Waldvogel (1996). For our particular problem (2), the Green's function $G\left(r, \tau, r^{\prime}, \tau^{\prime}\right)$ is defined as the solution of

$$
G_{\tau}=\frac{1}{2} \sigma^{2} r^{2 \beta} G_{r r}+\left(f(r, \tau)+\sigma(r, \tau) r^{\beta} q(r, \tau)\right) G_{r}-r G+\delta\left(r^{\prime}-r, \tau^{\prime}-\tau\right),
$$

where $\delta\left(r^{\prime}-r, \tau^{\prime}-\tau\right)$ is the Dirac delta function and $\tau^{\prime}=T-t^{\prime}$. The solution of equation (2) at any given time $(r, \tau)$ is given by

$$
P(r, \tau)=\int_{\Omega} G\left(r, \tau, r^{\prime}, \tau^{\prime}=0\right) \Phi\left(r^{\prime}, \tau^{\prime}=0\right) d r^{\prime}
$$

where $\Phi\left(r^{\prime}, \tau^{\prime}\right)$ represents the payoff function and $\Omega$ is the domain of $r$. For example, a zero coupon bond paying a principal amount of $\$ 1$ can be valued by solving

$$
P(r, \tau)=\int_{\Omega} G\left(r, \tau, r^{\prime}, \tau^{\prime}=0\right) d r^{\prime}
$$

A general algorithm for pricing semi-American callable bonds was presented in Section 2. In the context of this particular method, we:

1. Solve equation (7) from $T$ to $t_{c_{M}}$, with initial condition $K(r, \tau=0)=$ Principal $+C$ :

$$
K\left(r, \tau_{c_{M}}^{-}\right)=\int_{\Omega} G\left(r, \tau_{c_{M}}, r^{\prime}, \tau^{\prime}=0\right) K(r, \tau=0) d r^{\prime} .
$$

2. Add the coupon payment to the solution

$$
K\left(r, \tau_{c_{M}}^{+}\right)=K\left(r, \tau_{c_{M}}^{-}\right)+C .
$$

3. Solve for the present value of the call price

$$
\left[X\left(t_{c_{M}}\right)+C\right] P\left(r, \tau_{n_{M}}-\tau_{c_{M}}\right),
$$

from the call date $\tau_{c_{M}}$ to the notice date $\tau_{n_{M}}^{-}$

$$
P\left(r, \tau_{n_{M}}-\tau_{c_{M}}\right)=\int_{\Omega} G\left(r, \tau_{n_{M}}-\tau_{c_{M}}, r^{\prime}, \tau^{\prime}=0\right)\left[X\left(t_{c_{M}}\right)+C\right] d r^{\prime} .
$$

Also solve (7) with $K\left(r, \tau_{c_{M}}^{+}\right)$as initial condition for the same period. The solution an instant before the notice date is $K\left(r, \tau_{n_{M}}^{-}\right)$is given by

$$
K\left(r, \tau_{n_{M}}^{-}\right)=\int_{\Omega} G\left(r, \tau_{n_{M}}-\tau_{c_{M}}, r^{\prime}, \tau^{\prime}=0\right) K\left(r, \tau_{c_{M}}^{+}\right) d r^{\prime} .
$$

Then compute the break-even interest rate by solving

$$
\left[X\left(t_{c_{M}}\right)+C\right] P\left(r_{b}, \tau_{n_{M}}-\tau_{c_{M}}\right)-K\left(r_{b}, \tau_{n_{M}}^{-}\right)=0 .
$$

Apply either Method 1 or Method 2 to update the solution and solve equation (7) to the next coupon payment. 
4. Repeat steps 2-3 for the remaining call dates.

5. Solve back to $t_{0}$, adding coupon payments at remaining coupon dates along the way.

Of course, applying this algorithm requires that we know the Green's function $G$. This is only available in special cases. The two cases considered by Büttler and Waldvogel (1996) are the single factor models of Vasicek (1977) and CIR (1985) with constant parameters. The Green's functions for these two models have appeared in various papers in the literature (e.g. Jamshidian, 1987; Beaglehole and Tenney, 1991). In addition to a lack of generality, there are other potential deficiencies with this approach. When valuing semi-American callable bonds, we need a separate numerical integration for each coupon payment. With each integration introducing errors, it can become very difficult to obtain accurate solutions. Moreover, the method is not directly applicable to American type contracts. Since one of our objectives is to develop a general numerical PDE method for valuing a variety of contracts, we will use this Green's function approach to provide validation tests for our numerical PDE approach.

We will now describe a second technique for solving equation (2). This numerical PDE approach involves discretizing the equation using a finite volume method (Kröner, 1997). This is a powerful and flexible approach which is popular in the field of computational fluid dynamics. It is particularly useful for equations with convection-dominance. As we shall see, this is characteristic of interest rate models with mean-reversion. A detailed analysis of finite volume methods in the context of financial applications can be found in Zvan et al. (2000). Letting $P_{i}^{n}$ denote the value of the claim at interest rate node $r_{i}$ at time level $n$, the discretization can be written as

$$
\begin{aligned}
& A_{i}\left(\frac{P_{i}^{n+1}-P_{i}^{n}}{\Delta \tau}\right)= \\
& \gamma\left(\sum_{j \in \eta_{i}} \alpha_{i j}\left(P_{j}^{n+1}-P_{i}^{n+1}\right)+\sum_{j \in \eta_{i}} \overrightarrow{L_{i j}} \cdot \overrightarrow{V_{i}} P_{i j+\frac{1}{2}}^{n+1}-r_{i} A_{i} P_{i}^{n+1}\right)+ \\
& \quad(1-\gamma)\left(\sum_{j \in \eta_{i}} \alpha_{i j}\left(P_{j}^{n}-P_{i}^{n}\right)+\sum_{j \in \eta_{i}} \overrightarrow{L_{i j}} \cdot \vec{V}_{i} P_{i j+\frac{1}{2}}^{n}-r_{i} A_{i} P_{i}^{n}\right),
\end{aligned}
$$

where

$$
\begin{aligned}
A_{i} & =\frac{r_{i+1}-r_{i-1}}{2}, \\
\eta_{i} & =\{i+1, i-1\}, \\
\Delta \tau & =\tau^{n+1}-\tau^{n}, \\
\gamma & =\text { temporal weighting }(0 \leq \gamma \leq 1), \\
P_{i j+\frac{1}{2}}^{n+1} & =\text { value of } P \text { at the face between nodes } i \text { and } j, \\
\alpha_{i j} & =\frac{\sigma\left(r_{i}, \tau\right)^{2} r_{i}^{2 \beta}}{2\left|r_{j}-r_{i}\right|}, \\
\vec{V}_{i} & =-\left(f\left(r_{i}, \tau\right)+\sigma\left(r_{i}, \tau\right) q\left(r_{i}, \tau\right) r_{i}^{\beta}\right) \hat{\mathrm{i}}, \\
\overrightarrow{L_{i j}} & =\left\{\begin{array}{l}
-\hat{\mathrm{i}} \quad \text { if } j=i+1 \\
+\hat{\mathrm{i}} \quad \text { if } j=i-1
\end{array},\right. \\
\hat{\mathrm{i}} & =\text { unit vector in the positive } r \text { direction. }
\end{aligned}
$$


The temporal weighting parameter determines the type of method as follows: when $\gamma=1$ we have a fully implicit method, for $\gamma=1 / 2$ we have a Crank-Nicolson method, and for $\gamma=0$ we have a fully explicit method. Also, note that discretization (10) allows for a non-uniformly spaced grid. Numerical efficiency is enhanced by having a fine grid spacing near regions of interest (e.g. close to the current value of $r$ ) and a coarse spacing far away from such regions.

In order to mitigate the negative potential effects of convection-dominance, we use a van Leer flux limiter in (10) to define $P_{i j+\frac{1}{2}}$ (see Zvan, Forsyth, and Vetzal, 1998, for details). In the Appendix, we derive the conditions under which

$$
P_{i j+\frac{1}{2}}= \begin{cases}P_{i}^{n+1}+\frac{\phi\left(q_{i j+\frac{1}{2}}^{n+1}\right)}{2}\left(P_{j}^{n+1}-P_{i}^{n+1}\right) & \text { if } \overrightarrow{L_{i j}} \cdot \overrightarrow{V_{i}} \leq 0 \\ P_{j}^{n+1}+\frac{\phi\left(q_{j i+\frac{1}{2}}^{n+1}\right)}{2}\left(P_{i}^{n+1}-P_{j}^{n+1}\right) & \text { if } \overrightarrow{L_{i j}} \cdot \vec{V}_{i}>0\end{cases}
$$

with fully implicit temporal weighting on a non-uniform grid produces a positive coefficient discretization. This is useful in suppressing spurious oscillations arising from discontinuities. Here $\phi\left(q_{i j+\frac{1}{2}}^{n+1}\right)$ is the limiter function and

$$
q_{i j+\frac{1}{2}}=\frac{P_{i}^{n+1}-P_{2 u p i j}^{n+1}}{P_{j}^{n+1}-P_{i}^{n+1}} .
$$

The location of the second upstream point is given in Figure 3. In the fully implicit case $(\gamma=1)$, for discretization (10) we require

$$
1-\frac{\phi\left(q_{j i}^{n+1}\right)}{2} \geq 0 \text { and } \frac{\phi\left(q_{i j+\frac{1}{2}}^{n+1}\right)}{2 q_{i j+\frac{1}{2}}^{n+1}} \geq 0
$$

to ensure that we have a positive coefficient scheme. If a Crank-Nicolson method is used, in addition to conditions (12), we also have a condition on the allowed timestep size to ensure we still have a positive coefficient scheme (see Zvan et al. (2000)).

\section{Boundary Conditions}

To complete our discretization, we need to specify boundary conditions. In the Vasicek model, this is straightforward. In this model $r$ is assumed to follow a mean-reverting process of the form

$$
d r=\kappa(\theta-r) d t+\sigma d z
$$

where $\kappa$ denotes the speed of adjustment and $\theta$ is reversion level. Moreover, the market price of interest rate risk is specified as a constant $q(r, \tau)=q$. As a result, for this particular model equation (2) can be written

$$
P_{\tau}=\frac{1}{2} \sigma^{2} P_{r r}+(\kappa(\theta-r)+\sigma q) P_{r}-r P .
$$

In equation (13) the diffusion term is nonzero at $r=0$, so it is possible for $r$ to become negative. This means that the interest rate domain for this model is from $-\infty$ to $+\infty$. No boundary condition 
needs to be imposed to solve equation (14) as $|r| \rightarrow \infty$ since the first order term dominates here, corresponding to information flow away from the origin. This convection-dominance for large absolute values of $r$ means that flux limiting techniques should be used to alleviate possible problems with spurious oscillations. Intuitively, the reason why no boundary conditions are required is that even though the PDE (14) is formally parabolic, it behaves numerically like a hyperbolic equation for extreme absolute values of $r$.

In the single factor CIR model, the interest rate process is

$$
d r=\kappa(\theta-r) d t+\sigma \sqrt{r} d z .
$$

The market price of interest rate risk is specified as $q(r, \tau)=-\hat{q} r / \sigma \sqrt{r}$, where $\hat{q}$ is a constant, implying that in this case the PDE (2) takes the form

$$
P_{\tau}=\frac{1}{2} \sigma^{2} r P_{r r}+(\kappa(\theta-r)-\hat{q} r) P_{r}-r P .
$$

The domain of definition for this model is $r=[0, \infty)$. As $r \rightarrow \infty, P(\tau, r \rightarrow \infty)=0$. The situation as $r \rightarrow 0$ is somewhat more complicated, however.

Letting $r \rightarrow 0$ in equation (16), we obtain

$$
P_{\tau}=\kappa \theta P_{r}
$$

Assuming $\kappa \theta \geq 0$, equation (17) is a first order hyperbolic equation with outgoing characteristic. This suggests that no boundary condition should be required at $r=0$. However, at any finite (positive) distance from the origin, there is still a diffusion (parabolic) term in equation (16). Consequently, we expect that if the diffusion term tends to zero sufficiently rapidly as $r \rightarrow 0$, then equation (16) is essentially hyperbolic at $r=0$, so that no boundary condition is required. However, if the diffusion term does not tend to zero sufficiently rapidly, then equation (16) will require a boundary condition at $r=0$. This issue is resolved as a special case of results in Feller (1951). There it is shown that for the CIR one factor model (16), no boundary condition is required at $r=0$ if $2 \kappa \theta / \sigma^{2} \geq 1$.

From a financial point of view this is still not satisfactory. It does not make any sense to impose a condition at $r=0$ since this would imply that the price of a bond at $r=0$ can be arbitrarily specified. As such, the restriction $2 \kappa \theta / \sigma^{2} \geq 1$ seems artificial and unnecessary. It follows from the results of Oleinik and Radkevic (1973) that for any equation of the form

$$
P_{\tau}=a(r) P_{r r}+b(r) P_{r}+c(r) P
$$

no boundary condition is required at $r=0$ as long as $\lim _{r \rightarrow 0}\left(b(r)-a_{r}(r)\right) \geq 0$. Note that these conditions have been generalized in Houston et al. (2000). For the CIR model this is just our condition that $2 \kappa \theta / \sigma^{2} \geq 1$.

The conditions of Oleinik and Radkevic lead one to naturally consider a perturbed version of the CIR model, which we will call the $\epsilon$-CIR model. This is a stochastic model for the short term interest rate of the form

$$
d r=\kappa(\theta-r) d t+\sigma \sqrt{r^{1+\epsilon}} d z
$$

where $|\epsilon| \ll 1$. This $\epsilon$-CIR process is practically indistinguishable from the case $\epsilon=0$, for any $r>0$, for $|\epsilon|$ sufficiently small. To simplify notation, in the following we will assume that the market price of interest rate risk is zero (or, alternatively, that process (18) is specified under the 
risk-neutral measure). Using standard arguments, we obtain the following PDE for the price of an interest rate contingent claim

$$
P_{\tau}=\frac{1}{2} \sigma^{2} r^{1+\epsilon} P_{r r}+\kappa(\theta-r) P_{r}-r P .
$$

Since $\lim _{r \rightarrow 0}\left(\kappa(\theta-r)-\frac{1}{2} \sigma^{2}(1+\epsilon) r^{\epsilon}=\kappa \theta>0\right.$ we see that process (18) has the desirable property that no boundary is required at $r=0$ for the corresponding PDE when $\epsilon>0$. Indeed this property combined with the fact that it agrees with the CIR model as $\epsilon \rightarrow 0$ is the reason we consider this perturbed model in the first place.

However, for our purposes it is not enough to know that there is no boundary required at $r=0$ since we still need to know how to discretize (19) at $r=0$. A naive argument implies that this can be done by discretizing equation (17). However, because of the limiting procedures that we are using one needs to carefully determine if this is indeed the case. In order to check this we look at the power series solutions of the linear ordinary differential equations (ODEs) determined via separation of variables and then take the limit as $\epsilon \rightarrow 0$.

Let $\epsilon=1 / m$ with $m$ an integer. If $P(r, \tau)=y(r) w(\tau)$, then we obtain

$$
w_{\tau}=\lambda w,
$$

and

$$
\frac{1}{2} \sigma^{2} r^{1+1 / m} y_{r r}+\kappa(\theta-r) y_{r}-(r+\lambda) y=0,
$$

where $\lambda$ is independent of $r$ and $\tau$. We are interested in the behaviour of our solution as $r \rightarrow 0$ and so we investigate equation (20) by looking at series solutions. The change of variables $r=x^{m}$ gives

$$
\frac{1}{2} \frac{\sigma^{2}}{m^{2}} x^{3-m} y_{x x}+\left(\frac{1}{2} \frac{\sigma^{2}}{m^{2}} x^{2-m}(1-m)+\frac{\kappa \theta}{m} x^{1-m}-\frac{\kappa}{m} x\right) y_{x}-\left(x^{m}+\lambda\right) y=0 .
$$

In this case the point $x=0$ is an irregular singular point (Ritger and Rose, 1968), which implies that we need to look for series solutions of the form

$$
y(x)=x^{\alpha} \exp \left(b / x^{\zeta}\right) \sum_{i=0}^{\infty} a_{i} x^{i} .
$$

Here $\zeta$ corresponds to a slope of Newton's polygon (Davenport et al., 1989) and $b$ is the solution of the corresponding characteristic equation. Using standard techniques for solving linear ODEs with irregular singular points (Davenport et al., 1989) along with some rather tedious algebra, we obtain a general solution of the form $y(x)=C_{1} y_{1}(x)+C_{2} y_{2}(x)$, where

$$
\begin{aligned}
& y_{1}(x)=1+\frac{\lambda}{\kappa \theta} x^{m}+O\left(x^{2 m}\right), \\
& y_{2}(x)=x^{m+1} \exp \left(m \frac{2 \kappa \theta}{\sigma^{2} x}\right)\left[1+\left(\frac{m+1}{m}\right) \frac{\sigma^{2}}{2 \kappa \theta} x+O\left(x^{m+1}\right)\right] .
\end{aligned}
$$

Substitution back to the original variable via $x=r^{1 / m}$ gives $y(r)=C_{1} y_{1}(r)+C_{2} y_{2}(r)$, where

$$
\begin{aligned}
y_{1}(r) & =1+\frac{\lambda}{\kappa \theta} r+O\left(r^{2}\right), \\
y_{2}(r) & =r^{(m+1) / m} \exp \left(m \frac{2 \kappa \theta}{\sigma^{2}} r^{-1 / m}\right)\left[1+\left(\frac{m+1}{m}\right) \frac{\sigma^{2}}{2 \kappa \theta} r^{1 / m}+O\left(r^{1+1 / m}\right)\right] \\
& =r^{1+\epsilon} \exp \left(\frac{1}{\epsilon} \frac{2 \kappa \theta}{\sigma^{2}} r^{-\epsilon}\right)\left[1+(\epsilon+1) \frac{\sigma^{2}}{2 \kappa \theta} r^{\epsilon}+O\left(r^{1+\epsilon}\right)\right] .
\end{aligned}
$$


Note that, for any fixed $\epsilon>0$,

$$
\lim _{r \rightarrow 0} \overbrace{\left(r^{1+\epsilon} \exp \left(\frac{2 \kappa \theta}{\sigma^{2}} \frac{r^{-\epsilon}}{\epsilon}\right)\right)}^{I(r)}=\infty
$$

and hence $y_{2}(r)$ is unbounded at $r=0$. Consequently, if we require that the solution to equation (19) be bounded as $r \rightarrow 0$, we must exclude $y_{2}(r)$.

Consider now equation (17) and let $\hat{P}(r, \tau)=\hat{y}(r) \cdot w(\tau)$ be a solution of this PDE. Then we obtain the two equations

$$
\begin{aligned}
w_{\tau} & =\lambda w, \\
\kappa \theta \hat{y}_{r} & =\lambda \hat{y} .
\end{aligned}
$$

This implies that

$$
\hat{y}(r)=Q \exp \left(\frac{\lambda}{\kappa \theta} r\right)=Q\left(1+\frac{\lambda}{\kappa \theta} r+O\left(r^{2}\right)\right)
$$

for a constant $Q$. Therefore the bounded solution (24) agrees asymptotically as $r \rightarrow 0$ (up to $O\left(r^{2}\right)$ ) with the solution of equation (27). It then follows that in order to obtain convergence to the finite solution of (19) as $\epsilon \rightarrow 0$, it is only necessary to discretize equation (17) appropriately at $r=0$.

We can complete our discretization now as follows. In the fully implicit case $(\gamma=1)$, at $i=0$ (i.e. $r=0$ ), equation (10) becomes

$$
A_{0} \frac{P_{0}^{n+1}-P_{0}^{n}}{\Delta \tau}=\sum_{j \in \eta_{0}} \overrightarrow{L_{0 j}} \cdot \overrightarrow{V_{0}} P_{0 j+\frac{1}{2}}^{n+1} .
$$

Assuming that $\vec{V}_{0} \cdot \hat{\mathrm{i}}<0$, we obtain

$$
A_{0} \frac{P_{0}^{n+1}-P_{0}^{n}}{\Delta \tau}=\left|\vec{V}_{0}\right|\left[P_{1}^{n+1}+\frac{\phi\left(q_{10+\frac{1}{2}}^{n+1}\right)}{2}\left(P_{0}^{n+1}-P_{1}^{n+1}\right)\right]+P_{0}^{n+1}\left|\vec{V}_{0}\right|,
$$

where we have used pure upstream weighting for the outgoing flux at $r=0$. Equation (29) can be written

$$
P_{0}^{n+1}\left[1-\left|\vec{V}_{0}\right| \frac{\Delta \tau}{A_{0}} \frac{\phi\left(q_{10+\frac{1}{2}}^{n+1}\right)}{2}+\left|\vec{V}_{0}\right| \frac{\Delta \tau}{A_{0}}\right]=P_{0}^{n}+\left|\vec{V}_{0}\right| \frac{\Delta \tau}{A_{0}}\left[1-\frac{\phi\left(q_{10+\frac{1}{2}}^{n+1}\right)}{2}\right] P_{1}^{n+1}
$$

or

$$
P_{0}^{n+1}\left[1+\left|\vec{V}_{0}\right| \frac{\Delta \tau}{A_{0}}\left(1-\frac{\phi\left(q_{10+\frac{1}{2}}^{n+1}\right)}{2}\right)\right]=P_{0}^{n}+\left|\vec{V}_{0}\right| \frac{\Delta \tau}{A_{0}}\left[1-\frac{\phi\left(q_{10+\frac{1}{2}}^{n+1}\right)}{2}\right] P_{1}^{n+1}
$$




\begin{tabular}{|c|c|c|c|c|c|c|}
\hline & & \multicolumn{2}{|r|}{ Vasicek } & CIR & Year & Call Price \\
\hline Maturity $T$ & 20.172 years & $\kappa$ & 0.44178462 & 0.54958046 & $1-5$ & $\$ 1.000$ \\
\hline Coupon $C$ & $4.25 \%$ & $\sigma$ & 0.13264223 & 0.38757496 & 6 & $\$ 1.005$ \\
\hline Principal & $\$ 1.000$ & $\theta$ & 0.0348468515 & 0.0348468515 & 7 & $\$ 1.010$ \\
\hline Notice period & 0.1666 years & $q, \hat{q}$ & 0.21166329 & -0.40663675 & 8 & $\$ 1.015$ \\
\hline & & & & & $\begin{array}{c}9 \\
10\end{array}$ & $\begin{array}{l}\$ 1.020 \\
\$ 1.025\end{array}$ \\
\hline
\end{tabular}

TABLE 1: Input data used for the models considered. The data are as given in Büttler and Waldvogel (1996). Note that the years for the call prices run backwards in time, so for example the bond is callable at a price of \$1.025 ten years before maturity. Coupon payments are on an annual basis.

This will be a positive coefficient method. At $i=0$, if the usual limiter conditions are satisfied, it follows immediately from equation (31) that

$$
P_{0}^{n+1} \leq \max \left(P_{0}^{n}, P_{1}^{n+1}\right) \text { and } P_{0}^{n+1} \geq \min \left(P_{0}^{n}, P_{1}^{n+1}\right) .
$$

In the Appendix, we show that (for $\gamma=1$ )

$$
P_{i}^{n+1} \leq \max \left(P_{j \in \eta_{i}}^{n+1}, P_{i}^{n}\right) \text { for } i \neq 0
$$

and

$$
P_{i}^{n+1} \geq \frac{1}{1+r_{i} \Delta \tau} \min \left(P_{j \in \eta_{i}}^{n+1}, P_{i}^{n}\right) \text { for } i \neq 0 .
$$

In practice, we truncate the computational domain from $[0, \infty)$ to $\left[0, r_{\max }\right)$, where $r_{\max }$ is chosen to be sufficiently large that computed values are not appreciably affected by the upper boundary. Note that $\max _{i}\left(P_{i}^{0}\right)=$ Principal $+C$. We then have

$$
0 \leq P_{i}^{n+1} \leq \text { Principal }+ \text { number of coupon payments } \times C \quad \forall i, n,
$$

so that discretization (10) produces stable bounded solutions.

It is straightforward to consider the case where $\gamma=\frac{1}{2}$ (Crank-Nicolson timestepping). As well as the usual flux limiter conditions, we also obtain a condition on the timestep size in order to obtain a positive coefficient discretization. This derivation is algebraically tedious, and not particularly illuminating, so we omit the details here.

\section{Results}

In order to facilitate comparison with the Green's function approach of Büttler and Waldvogel (1996), we concentrate on the Vasicek and CIR models. Unless stated otherwise, we use Büttler and Waldvogel's input data (summarized in Table 1$)$. Crank-Nicolson time weighting $(\gamma=1 / 2$ in discretization (10)) was used. The discrete system of equations is nonlinear due to the use of a flux limiter, and was solved with Newton iteration with a convergence tolerance of $10^{-5}$. The Jacobian was solved using a direct method. We also used the automatic timestep selector defined by Johnson (1987); an initial timestep is given and the next timestep is computed according to

$$
\frac{\tau^{n+2}-\tau^{n+1}}{\tau^{n+1}-\tau^{n}}=\frac{d}{\max _{i} \frac{\left|P_{i}^{n+1}-P_{i}^{n}\right|}{\max \left(1,\left|P_{i}^{n}\right|\right)},}
$$


where $d$ specifies the maximum relative change allowed. Recall that for the Vasicek model, the PDE is defined on the domain $r \in(-\infty, \infty)$, while for the CIR model $r \in[0,-\infty)$. In practice, the computational domain is truncated to $r \in\left[-r_{\max },+r_{\max }\right]$ for the Vasicek model and $r \in\left[0,+r_{\max }\right]$ for the CIR model. All the tests were run on a Sun Ultra Sparc and the CPU times are reported in seconds.

\subsection{The Effects of a Discontinuity in the Solution}

We begin by considering the issues raised by the presence of a discontinuity in the solution. We will consider here only the CIR model, since similar conclusions can be deduced for the Vasicek model. Recall from Section 2 that using Method 2 to update the numerical solution will introduce a discontinuity at each call date. This can drastically reduce the accuracy of the discretization scheme unless certain precautions are taken.

It is well-known that Crank-Nicolson schemes are unconditionally stable. However, this property does not prevent spurious oscillations from forming unless certain conditions are met (Zvan, Vetzal, and Forsyth, 1998). Figures 4(a) and 4(b) illustrate what can happen when the first timestep chosen is too large. Even though the plot for the callable bond price in Figure 4(a) looks reasonable, there are slight oscillations present which become significantly amplified in the "delta" (i.e. $\partial P / \partial r$ ) of the callable bond (Figure 4(b)). Since delta is important for hedging purposes, methods should be used which do not permit these oscillations to develop.

To explore this effect further, consider the following extreme example. The underlying asset is a 6 year zero-coupon bond that can only be called 1 year before maturity and the notice period is 4.5 years. Apart from this, we use the CIR interest rate model parameters from Table 1. This contract is quite unrealistic, but it serves to illustrate numerical difficulties in an extreme case. Figures 5(a) and 5(b) show oscillations in the solution profile for the bond price that again are amplified in the bond delta.

One way to avoid the oscillations is to use a fully implicit method for a small number of timesteps after any discontinuities arise and Crank-Nicolson thereafter. This technique has been described by Rannacher (1984). It restores quadratic convergence, even in the presence of discontinuities. We applied this method using two fully implicit timesteps after the call date to the extreme example from Figure 5, and obtained the results shown in Figure 6. Even in this extreme case, the oscillations have vanished. Returning to the more realistic callable bond described in Table 1, as we would expect the use of the Rannacher technique removes the oscillations in this case as well (see Figure 7).

In order to obtain quadratic convergence in the presence of discontinuities, Rannacher (1984) suggests that the actual initial condition should be replaced by its $L_{2}$ projection onto the space of basis functions. We experimented with this smoothing method (projection onto linear basis functions), but did not notice any difference between smoothed and non-smoothed initial conditions. We note that a variable timestepping algorithm is used here, unlike the constant timestep used in Rannacher (1984). Since we always start with a very small timestep after the call date, we believe that this explains our observations.

It might be supposed that the discontinuity will reduce the solution's accuracy. To mitigate this possible problem, we added points around the discontinuity by creating an automatic refinement mechanism as follows. First, find the break-even interest rate $r_{b}$, i.e. $r_{b} \in\left[r_{i}, r_{i+1}\right]$. Then uniformly add $n$ nodes in $\left[r_{i-1}, r_{i+2}\right]$, and interpolate the solution onto this locally refined grid. The breakeven value $r_{b}$ is recomputed. Since $r_{b}$ changes over time, it would be computationally expensive to keep these extra points. Thus, each time $r_{b}$ is computed the grid is brought back to its original state and new nodes are added only near the current $r_{b}$. Experiments indicated that since the 
discontinuities were quite small, accuracy of the solution was not noticeably improved using adaptive mesh refinement (if Rannacher's method was used). Note that using local refinement will make oscillations worse, since $\Delta t / \Delta r^{2}$ will be larger. Consequently, all examples presented in the following used a non-adaptive grid combined with the Rannacher technique.

\subsection{Convergence \& Validation}

We validate our finite volume approach by demonstrating the convergence of prices for the Vasicek and CIR models. We consider different interest rate domains, and for each domain we double the number of points three times. For each test, as we double the number of points we cut both the initial timestep and the maximum relative change $d$ (as in equation (35)) in half. In each case we computed the price of the callable bond using both Method 1 and Method 2 as described in Section 2. Table 2 contains the results.

The results demonstrate that our approach can accurately value callable bonds in a reasonable computational time when we consider a sufficiently large domain. In particular, we observe that a computational domain with $r \in[-1,1]$ and 680 points is accurate to about five figures for the Vasicek model. We will use this domain and mesh/timestep parameters in subsequent Vasicek examples. Similarly, Table 2 demonstrates that a computational domain of $r \in[0,2.5]$ and 306 nodes gives results correct to about four figures for the CIR model. This domain and mesh/timestep parameters will be used in subsequent CIR examples.

In Table 3, we compare the results computed in this work using a numerical PDE approach and Method 1 to the results in Büttler and Waldvogel (1996), where the semi-analytical Green's function technique was used. In the case of the CIR model, we obtain excellent agreement for both the straight bond and the callable bond. However, for the Vasicek model, the results in Büttler and Waldvogel (1996) and in this work agree only in the case of the straight bond. For the callable bond, our results do not agree with those of Büttler and Waldvogel (1996).

As an additional check on the Vasicek results, we computed the price of the callable bond (for both Method 1 and Method 2), using the Green's function technique described in Section 3. We experimented with different integration rules and found that the composite Simpson's rule was the most effective (the solutions were accurate to 4-5 digits). Convergence testing is summarized in Table 4. Note that we used the same interest rate domains as in Table 2. Comparing Table 4 with the value of a callable bond at $r=.05$ in Table 3, we see that for the same level of accuracy we needed about 1000 nodes for the Green's function integration, but only about 330 nodes for the finite volume method.

Table 5 summarizes our results for the Vasicek model, using both Method 1 and Method 2. The table shows that both the Green's function and the numerical PDE approach are in excellent agreement with each other for both methods. However, the results for Method 1 do not correspond very closely with those in Büttler and Waldvogel (1996). We are unable to account for this disagreement. There may be some subtle misunderstanding on our part concerning the input data and parameters used in Büttler and Waldvogel (1996). Nevertheless, it would appear that for well defined input data, the numerical PDE approach converges rapidly to the correct solution, for both the Vasicek and the CIR models.

\subsection{Some Further Results}

We computed the value of a callable bond for both the Vasicek and CIR models using different numbers of call dates (up to ten), with other data as in Table 1. With a single call date, the bond is callable at the final coupon payment prior to maturity; with two call dates, it can be called at 


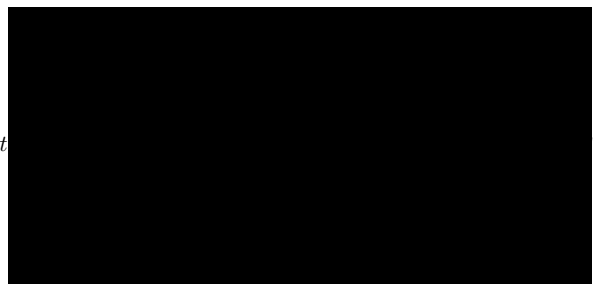

FIGURE 1: Time line representation of a European callable bond. $T$ is the maturity date, $t_{0}$ is the present time, $\tau_{0}=T-t_{0}$ is the length of time until maturity, and the coupon dates prior to $T$ are $t_{c_{i}}, i=1, \ldots, M$. The call date is $t_{c_{M}}$, and the notice date is $t_{n_{M}}$. In the backward direction, the time immediately before (after) the notice date is $\tau_{n_{M}}^{-}\left(\tau_{n_{M}}^{+}\right)$. 


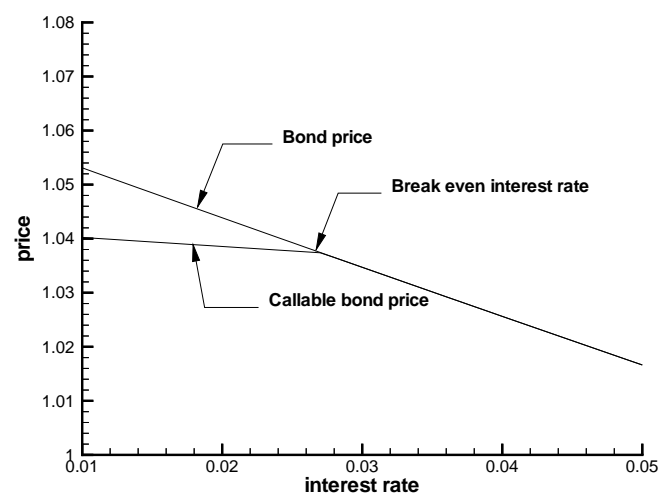

(a) The solution for the callable bond if the price is updated using Method 1, i.e. at the notice date.

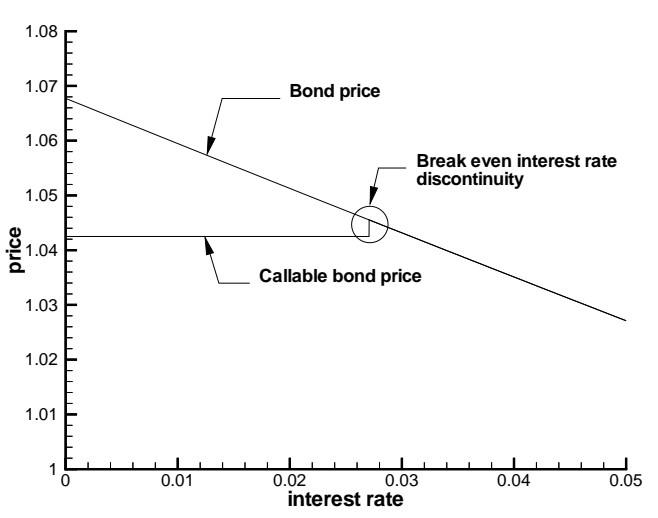

(b) The solution for the callable bond if the price is updated using Method 2, i.e. at the call date.

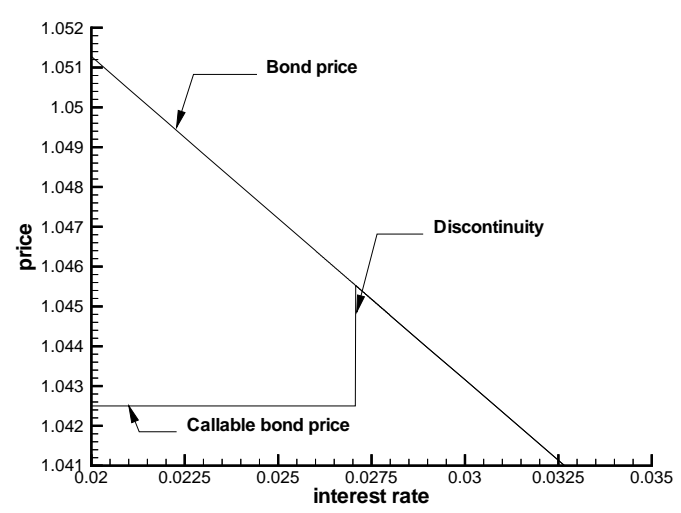

(c) Zoom of Figure 2(b) around the discontinuity.

FIGURE 2: Updating the solution for a European callable bond using Method 1 and Method 2. The case shown is for the Vasicek model, using the input data defined in Table 1.

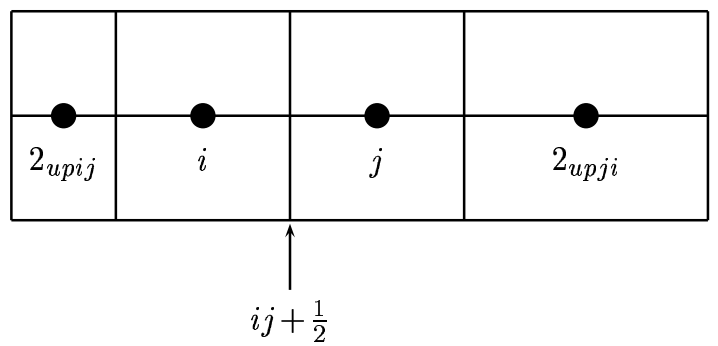

FiguRE 3: Schematic representation of the finite volume method. 


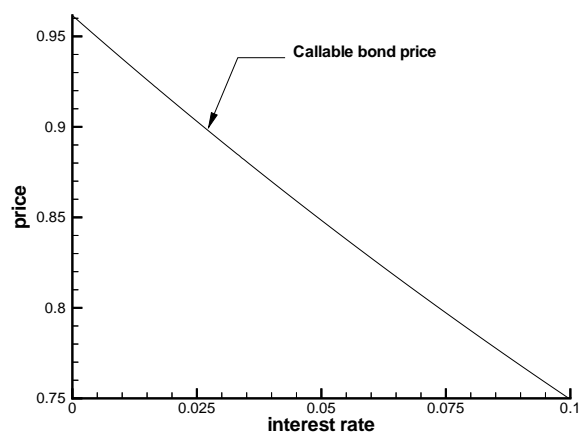

(a) Callable bond price.

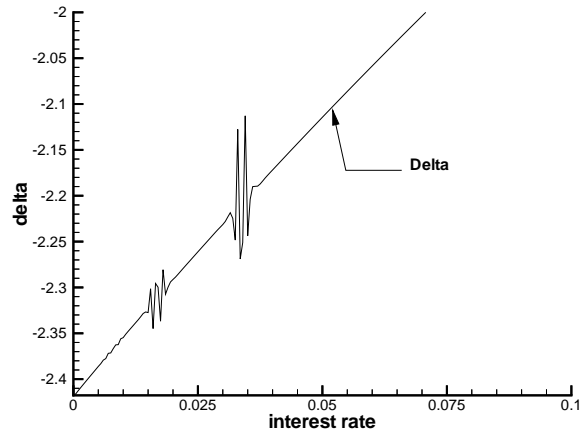

(b) Callable bond delta.

FIGURE 4: Results for finite volume method with 306 points for the interest rate domain $[0,2.5]$ with initial timestep 0.002 and maximum relative change allowed $d=0.02$. Results are for the CIR model with input data from Table 1. Method 2 was used to update the solution at the call dates.

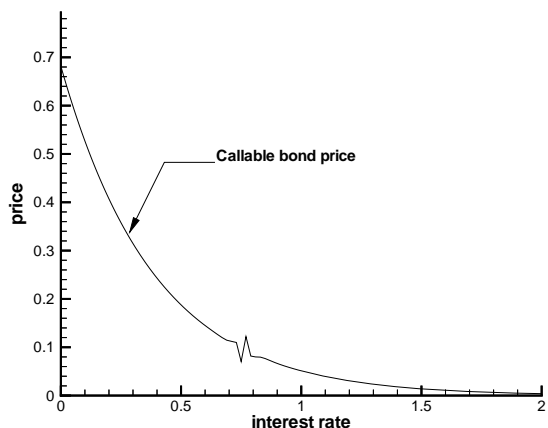

(a) Callable bond price with extreme notice period.

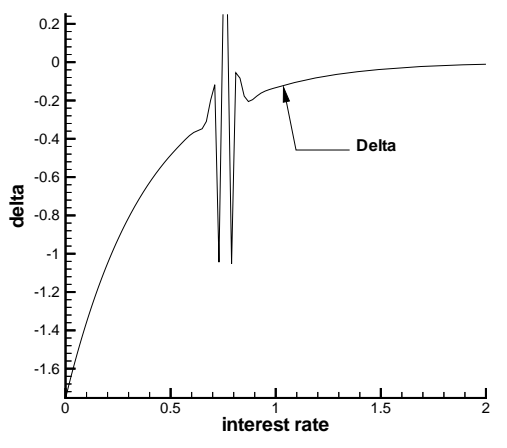

(b) Callable bond delta with extreme notice period.

Figure 5: Price and delta of a 6 year callable bond that can be called 1 year before the maturity date. The notice period is 4.5 years. The results are for the finite volume method with 306 points for the interest rate domain $[0,2.5]$ with initial timestep 0.2 and maximum relative change allowed $d=0.2$. Results are for the CIR model using Method 2 to update the solution at the call date. 


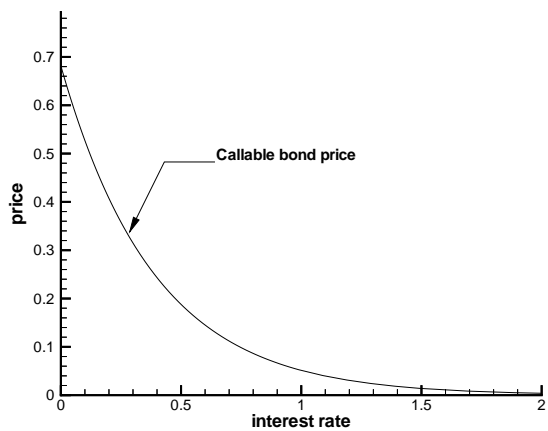

(a) Callable bond price with extreme notice period.

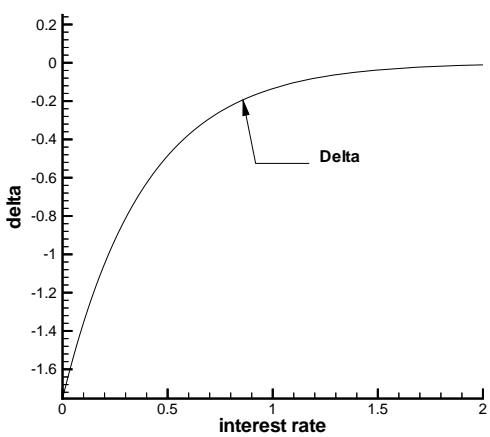

(b) Callable bond delta with extreme notice period.

Figure 6: Price and delta of a 6 year callable bond that can be called 1 year before the maturity date. The notice period is 4.5 years. The results are for the finite volume method with 306 points for the interest rate domain $[0,2.5]$ with initial timestep 0.2 and maximum relative change allowed $d=0.2$. Results are for the CIR model using Method 2 to update the solution at the call date. The method of Rannacher (1984) was used with two fully implicit timesteps immediately after the call date, and Crank-Nicolson otherwise.

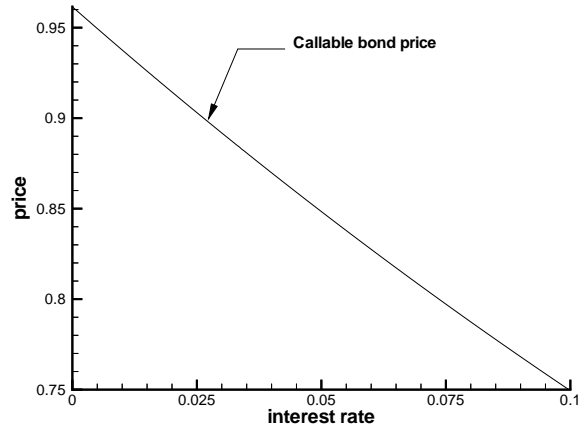

(a) Callable bond price.

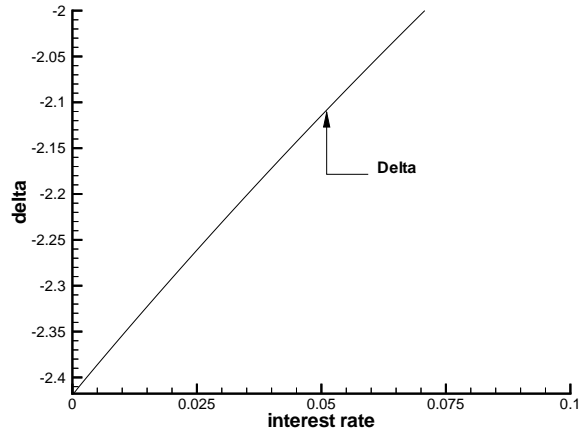

(b) Callable bond delta.

FiguRE 7: Results for finite volume method with 306 points for the interest rate domain $[0,2.5]$ with initial timestep 0.002 and maximum relative change allowed $d=0.02$. Results are for the CIR model with input data from Table 1. Method 2 was used to update the solution at the call dates. The method of Rannacher (1984) was used with two fully implicit timesteps immediately after the call date, and Crank-Nicolson otherwise. 


\begin{tabular}{|c|c|c|c|c|c|c|}
\hline & & & \multicolumn{2}{|c|}{ Method 1} & \multicolumn{2}{|c|}{ Method 2} \\
\hline $\begin{array}{l}\text { Number of } \\
\text { Grid Points }\end{array}$ & $\begin{array}{c}\text { Initial } \\
\text { Time Step }\end{array}$ & $\begin{array}{l}\text { Maximum } \\
\text { Change } d\end{array}$ & $\begin{array}{c}\text { Computed } \\
\text { Value }\end{array}$ & $\begin{array}{l}\text { CPU } \\
\text { (sec.) }\end{array}$ & $\begin{array}{c}\text { Computed } \\
\text { Value }\end{array}$ & $\begin{array}{l}\mathrm{CPU} \\
\text { (sec.) }\end{array}$ \\
\hline \multicolumn{7}{|c|}{ Vasicek Model } \\
\hline \multicolumn{7}{|c|}{$r \in[-0.5,0.5]$} \\
\hline 120 & $8.10^{-3}$ & 0.08 & 0.76563 & 1.22 & 0.75912 & 1.14 \\
\hline 280 & $4.10^{-3}$ & 0.04 & 0.76567 & 2.72 & 0.75912 & 2.62 \\
\hline 580 & $2.10^{-3}$ & 0.02 & 0.76596 & 5.78 & 0.75913 & 5.41 \\
\hline 1200 & $1.10^{-3}$ & 0.01 & 0.76568 & 13.56 & 0.75914 & 12.07 \\
\hline \multicolumn{7}{|c|}{$r \in[-1.0,1.0]$} \\
\hline 140 & $8.10^{-3}$ & 0.08 & 0.77846 & 1.54 & 0.77184 & 1.40 \\
\hline 330 & $4.10^{-3}$ & 0.04 & 0.77864 & 3.24 & 0.77184 & 3.16 \\
\hline 680 & $2.10^{-3}$ & 0.02 & 0.77868 & 6.92 & 0.77203 & 6.37 \\
\hline 1400 & $1.10^{-3}$ & 0.01 & 0.77869 & 14.96 & 0.77204 & 13.87 \\
\hline \multicolumn{7}{|c|}{$r \in[-1.5,1.5]$} \\
\hline 150 & $8.10^{-3}$ & 0.08 & 0.77846 & 1.62 & 0.77184 & 1.48 \\
\hline 350 & $4.10^{-3}$ & 0.04 & 0.77864 & 3.42 & 0.77198 & 3.17 \\
\hline 730 & $2.10^{-3}$ & 0.02 & 0.77868 & 6.97 & 0.77203 & 6.80 \\
\hline 1500 & $1.10^{-3}$ & 0.01 & 0.77869 & 16.36 & 0.77204 & 15.06 \\
\hline \multicolumn{7}{|c|}{ CIR Model } \\
\hline \multicolumn{7}{|c|}{$r \in[0.0,1.5]$} \\
\hline 81 & $8.10^{-3}$ & 0.08 & 0.85051 & 0.63 & 0.84913 & 0.63 \\
\hline 161 & $4.10^{-3}$ & 0.04 & 0.84978 & 1.30 & 0.84841 & 1.16 \\
\hline 301 & $2.10^{-3}$ & 0.02 & 0.84972 & 2.29 & 0.84830 & 2.30 \\
\hline 621 & $1.10^{-3}$ & 0.01 & 0.84972 & 4.98 & 0.84829 & 5.16 \\
\hline \multicolumn{7}{|c|}{$r \in[0.0,2.5]$} \\
\hline 86 & $8.10^{-3}$ & 0.08 & 0.85060 & 0.65 & 0.84920 & 0.67 \\
\hline 166 & $4.10^{-3}$ & 0.04 & 0.84987 & 1.49 & 0.84847 & 1.25 \\
\hline 306 & $2.10^{-3}$ & 0.02 & 0.84980 & 2.37 & 0.84837 & 2.29 \\
\hline 626 & $1.10^{-3}$ & 0.01 & 0.84980 & 5.06 & 0.84835 & 5.08 \\
\hline \multicolumn{7}{|c|}{$r \in[0.0,3.5]$} \\
\hline 87 & $8.10^{-3}$ & 0.08 & 0.85060 & 0.96 & 0.84920 & 0.65 \\
\hline 167 & $4.10^{-3}$ & 0.04 & 0.84987 & 1.95 & 0.84847 & 1.20 \\
\hline 307 & $2.10^{-3}$ & 0.02 & 0.84980 & 2.38 & 0.84837 & 2.28 \\
\hline 627 & $1.10^{-3}$ & 0.01 & 0.84980 & 5.07 & 0.84835 & 5.14 \\
\hline
\end{tabular}

TABLE 2: Convergence results for the Vasicek and CIR models. The input data is from Table 1. The computed values are given for $r=.05$. 


\begin{tabular}{|c|c|c|c|c|}
\hline & \multicolumn{2}{|c|}{ This work } & \multicolumn{2}{|c|}{ Büttler and Waldvogel (1996) } \\
\hline$r$ & Straight Bond & Callable Bond & Straight Bond & Callable Bond \\
\hline \multicolumn{5}{|c|}{ Vasicek Model } \\
\hline 0.01 & 0.92739 & 0.84282 & 0.9274 & 0.8556 \\
\hline 0.02 & 0.90892 & 0.82627 & 0.9089 & 0.8338 \\
\hline 0.03 & 0.89084 & 0.81007 & 0.8908 & 0.8223 \\
\hline 0.04 & 0.87315 & 0.79420 & 0.8731 & 0.8062 \\
\hline 0.05 & 0.85583 & 0.77868 & 0.8558 & 0.7904 \\
\hline 0.06 & 0.83887 & 0.76348 & 0.8389 & 0.7749 \\
\hline 0.07 & 0.82228 & 0.74860 & 0.8223 & 0.7598 \\
\hline 0.08 & 0.80604 & 0.73403 & 0.8060 & 0.7450 \\
\hline 0.09 & 0.79014 & 0.71977 & 0.7901 & 0.7305 \\
\hline 0.10 & 0.77458 & 0.70578 & 0.7746 & 0.7163 \\
\hline 0.11 & 0.75935 & 0.69214 & 0.7594 & 0.7024 \\
\hline 0.12 & 0.74444 & 0.67875 & 0.7445 & 0.6888 \\
\hline 0.13 & 0.72985 & 0.66565 & 0.7299 & 0.6755 \\
\hline 0.14 & 0.71556 & 0.65283 & 0.7156 & 0.6625 \\
\hline 0.15 & 0.70158 & 0.64027 & 0.7016 & 0.6497 \\
\hline 0.16 & 0.68789 & 0.62798 & 0.6879 & 0.6372 \\
\hline 0.17 & 0.67450 & 0.61594 & 0.6745 & 0.6250 \\
\hline 0.18 & 0.66138 & 0.60416 & 0.6614 & 0.6130 \\
\hline 0.19 & 0.64854 & 0.59262 & 0.6486 & 0.6013 \\
\hline 0.20 & 0.63597 & 0.58132 & 0.6360 & 0.5898 \\
\hline \multicolumn{5}{|c|}{ CIR Model } \\
\hline 0.01 & 0.95527 & 0.93926 & 0.9552 & 0.9392 \\
\hline 0.02 & 0.93155 & 0.91598 & 0.9315 & 0.9159 \\
\hline 0.03 & 0.90846 & 0.89333 & 0.9084 & 0.8933 \\
\hline 0.04 & 0.88599 & 0.87127 & 0.8859 & 0.8712 \\
\hline 0.05 & 0.86411 & 0.84980 & 0.8641 & 0.8498 \\
\hline 0.06 & 0.84281 & 0.82890 & 0.8428 & 0.8289 \\
\hline 0.07 & 0.82207 & 0.80855 & 0.8220 & 0.8085 \\
\hline 0.08 & 0.80188 & 0.78874 & 0.8018 & 0.7887 \\
\hline 0.09 & 0.78223 & 0.76945 & 0.7822 & 0.7694 \\
\hline 0.10 & 0.76309 & 0.75067 & 0.7631 & 0.7507 \\
\hline 0.11 & 0.74446 & 0.73238 & 0.7444 & 0.7324 \\
\hline 0.12 & 0.72633 & 0.71458 & 0.7263 & 0.7146 \\
\hline 0.13 & 0.70867 & 0.69725 & 0.7086 & 0.6973 \\
\hline 0.14 & 0.69147 & 0.68038 & 0.6915 & 0.6804 \\
\hline 0.15 & 0.67473 & 0.66394 & 0.6747 & 0.6640 \\
\hline 0.16 & 0.65844 & 0.64795 & 0.6584 & 0.6480 \\
\hline 0.17 & 0.64257 & 0.63237 & 0.6426 & 0.6324 \\
\hline 0.18 & 0.62711 & 0.61720 & 0.6271 & 0.6172 \\
\hline 0.19 & 0.61207 & 0.60243 & 0.6121 & 0.6025 \\
\hline 0.20 & 0.59742 & 0.58805 & 0.5974 & 0.5881 \\
\hline
\end{tabular}

TABLE 3: Comparison of our results with those of Büttler and Waldvogel (1996) using input data from Table 1. 


\begin{tabular}{|r|r|r|r|r|}
\hline \multicolumn{5}{|c|}{ Vasicek Model (Green's Function Approach) } \\
\hline Number of & \multicolumn{2}{|c|}{ Method 1 } & \multicolumn{2}{c|}{ Method 2 } \\
\cline { 2 - 5 } Grid Points & Value & CPU (sec.) & Value & CPU (sec.) \\
\hline \multicolumn{5}{|c|}{$r \in[-0.5,0.5]$} \\
\hline 100 & 0.77542 & 1.93 & 0.76826 & 2.78 \\
200 & 0.78329 & 7.60 & 0.76886 & 11.1 \\
500 & 0.77580 & 47.4 & 0.76916 & 69.3 \\
1000 & 0.77588 & 189.9 & 0.76922 & 276.9 \\
\hline \multicolumn{5}{|c|}{$r \in[-1.0,1.0]$} \\
\hline 200 & 0.77908 & 7.66 & 0.77190 & 11.1 \\
400 & 0.78647 & 30.5 & 0.77971 & 44.5 \\
1000 & 0.77870 & 190.6 & 0.77205 & 278.0 \\
2000 & 0.77870 & 763.3 & 0.77202 & 1112.4 \\
\hline \multicolumn{5}{|c}{$r \in[-1.5,1.5]$} \\
\hline 300 & 0.77907 & 17.4 & 0.77189 & 25.2 \\
600 & 0.77878 & 69.3 & 0.77205 & 101.2 \\
1500 & 0.77870 & 432.1 & 0.77202 & 629.2 \\
2400 & 0.77870 & 1734.9 & 0.77202 & 2520.5 \\
\hline
\end{tabular}

TABLE 4: Convergence tests for the Vasicek model using the Green's function approach of Büttler and Waldvogel (1996). The input data used is contained in Table 1. Results are for $r=.05$.

\begin{tabular}{|c|c|c|c|c|c|}
\cline { 2 - 5 } \multicolumn{1}{c|}{} & \multicolumn{3}{c|}{ This work } & Büttler and \\
Waldvogel \\
$r$ & \multicolumn{2}{|c|}{ Method 1 } & \multicolumn{2}{c|}{ Method 2 } & $(1996)$ \\
\cline { 2 - 5 } 0.01 & Finite Volume & Green's Function & Finite Volume & Green's Function & 0.8556 \\
0.02 & 0.84282 & 0.84285 & 0.83555 & 0.83556 & 0.8338 \\
0.03 & 0.81007 & 0.82630 & 0.81916 & 0.81917 & 0.8223 \\
0.04 & 0.79420 & 0.81009 & 0.80311 & 0.80313 & 0.8062 \\
0.05 & 0.77868 & 0.79423 & 0.78741 & 0.78743 & 0.7904 \\
0.06 & 0.76348 & 0.76350 & 0.77203 & 0.77205 & 0.7749 \\
0.07 & 0.74860 & 0.74862 & 0.75698 & 0.75700 & 0.7598 \\
0.08 & 0.73403 & 0.73405 & 0.74225 & 0.74226 & 0.7450 \\
0.09 & 0.71977 & 0.71979 & 0.71370 & 0.72784 & 0.7305 \\
0.10 & 0.70578 & 0.70583 & 0.69987 & 0.71372 & 0.7163 \\
0.11 & 0.69214 & 0.69216 & 0.68634 & 0.68635 & 0.7024 \\
0.12 & 0.67875 & 0.67878 & 0.67308 & 0.67310 & 0.6888 \\
0.13 & 0.66565 & 0.66568 & 0.66011 & 0.66013 & 0.6755 \\
0.14 & 0.65283 & 0.65285 & 0.64741 & 0.64743 & 0.6625 \\
0.15 & 0.64027 & 0.64030 & 0.63497 & 0.63499 & 0.6497 \\
0.16 & 0.62798 & 0.62800 & 0.62280 & 0.62282 & 0.6372 \\
0.17 & 0.61594 & 0.61597 & 0.61088 & 0.61090 & 0.6250 \\
0.18 & 0.60416 & 0.60418 & 0.59921 & 0.59923 & 0.6130 \\
0.19 & 0.59262 & 0.59264 & 0.58778 & 0.58780 & 0.6013 \\
0.20 & 0.58132 & 0.58135 & 0.57659 & 0.57661 & 0.5898 \\
\hline
\end{tabular}

TABLE 5: Comparison of finite volume approach with our implementation of Büttler and Waldvogel (1996)'s Green's function method and the results reported in Büttler and Waldvogel (1996). The input data used is contained in Table 1. 


\begin{tabular}{|c|c|c|c|c|c|c|}
\cline { 2 - 7 } \multicolumn{1}{c|}{} & \multicolumn{3}{c|}{ Vasicek } & \multicolumn{3}{c|}{ CIR } \\
Number of & $\begin{array}{c}\text { Break-even } \\
\text { Call Dates }\end{array}$ & Value & $\begin{array}{c}\text { CPU } \\
\text { Interest Rate }\end{array}$ & $\begin{array}{c}\text { Break-even } \\
\text { Interest Rate }\end{array}$ & Value & $\begin{array}{c}\text { CPU } \\
\text { (sec.) }\end{array}$ \\
\hline 1 & 0.02707322 & 0.84328 & 4.01 & 0.03389193 & 0.85838 & 1.93 \\
2 & -0.01013280 & 0.83244 & 4.31 & 0.01792222 & 0.85420 & 2.10 \\
3 & -0.03657688 & 0.82297 & 4.55 & 0.00978074 & 0.85155 & 2.35 \\
4 & -0.05703286 & 0.81456 & 4.85 & 0.00487097 & 0.85019 & 2.38 \\
5 & -0.07352697 & 0.80696 & 5.10 & 0.00156474 & 0.84980 & 2.62 \\
6 & -0.09102469 & 0.80034 & 5.33 & n.a. & 0.84980 & 2.81 \\
7 & -0.10484371 & 0.79433 & 5.57 & n.a. & 0.84980 & 2.83 \\
8 & -0.11656572 & 0.78877 & 5.90 & n.a. & 0.84980 & 2.85 \\
9 & -0.12673856 & 0.78358 & 6.16 & n.a. & 0.84980 & 2.87 \\
10 & -0.13569428 & 0.77868 & 6.48 & n.a. & 0.84980 & 2.91 \\
\hline
\end{tabular}

TABLE 6: Callable bond values and break-even interest rates for the Vasicek and CIR models, with different number of call dates. The solution is updated using Method 1 to facilitate comparison with results reported in Büttler and Waldvogel (1996). The break-even interest rates are for the first call date (going forward in time). The values are reported for $r=.05$.

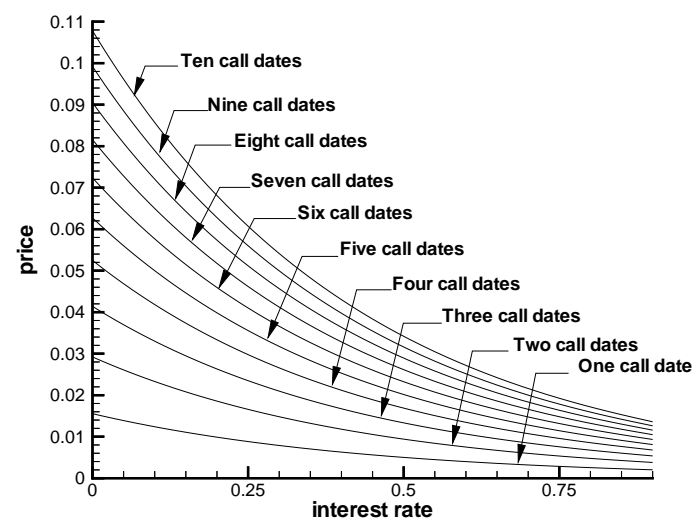

(a) Vasicek model.

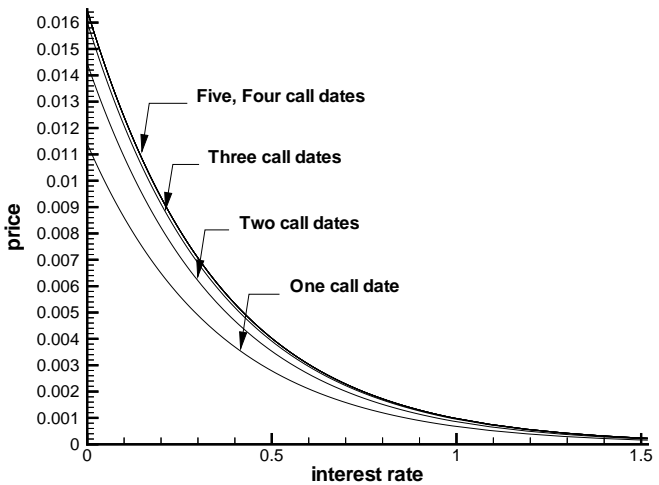

(b) CIR model.

Figure 8: Value of embedded call option for various numbers of call dates. The input data are given in Table 1.

the last two coupon payments before $T$, and so on. The results are shown in Table 6 . Intuitively, we expect that as the number of call dates increases, the value of the callable bond will decrease. In other words, the value of the embedded call option,

$$
\text { Embedded Option }=P_{\text {bond }}-P_{\text {callable bond }},
$$

increases with the number of call dates. This is illustrated in Figure 8. We used Method 1 to model the call policy in order to have results comparable with Büttler and Waldvogel (1996). As can be seen in Table 6, we obtain negative break-even interest rates for the Vasicek model. For the CIR model, there is no break-even interest rate when there are more than five call dates. In other words, it is not optimal to call the bond for any value of $r$ before the fifth coupon date prior 


\begin{tabular}{|c|c|c|c|c|}
\cline { 2 - 5 } \multicolumn{1}{c|}{} & \multicolumn{2}{c|}{ Vasicek } & \multicolumn{2}{c|}{ CIR } \\
\hline $\begin{array}{c}\text { Number of } \\
\text { Call Dates }\end{array}$ & Method 1 & Method 2 & Method 1 & Method 2 \\
\hline 1 & 0.84328 & 0.84219 & 0.85838 & 0.85780 \\
2 & 0.83244 & 0.83043 & 0.85420 & 0.85309 \\
3 & 0.82297 & 0.82017 & 0.85155 & 0.85002 \\
4 & 0.81456 & 0.81106 & 0.85019 & 0.84837 \\
5 & 0.80696 & 0.80284 & 0.84980 & 0.84837 \\
6 & 0.80034 & 0.79566 & 0.84980 & 0.84837 \\
7 & 0.79433 & 0.78913 & 0.84980 & 0.84837 \\
8 & 0.78877 & 0.78307 & 0.84980 & 0.84837 \\
9 & 0.78358 & 0.77739 & 0.84980 & 0.84837 \\
10 & 0.77868 & 0.77203 & 0.84980 & 0.84837 \\
\hline
\end{tabular}

TABle 7: Comparison of Method 1 vs. Method 2 for computing the value of a callable bond for various numbers of call dates. Values are given for $r=.05$.

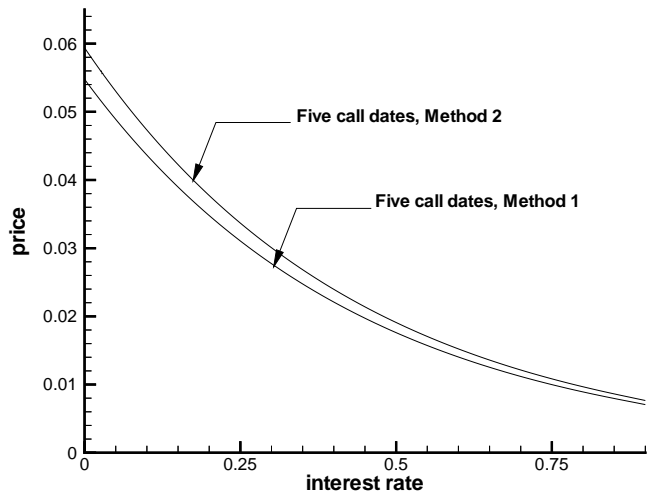

(a) Five call dates, Vasicek model.

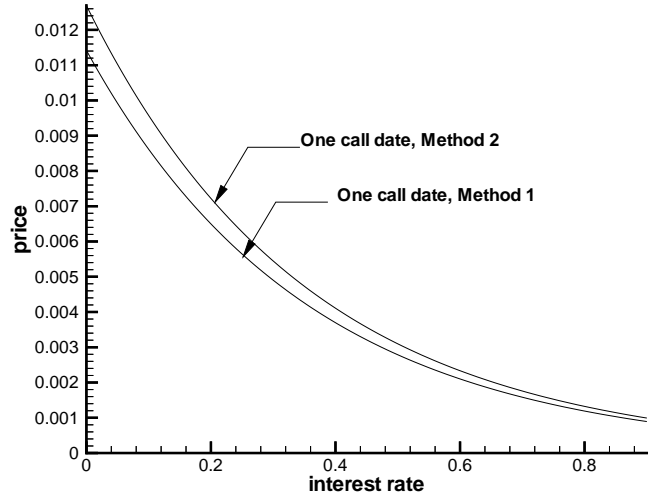

(b) One call date, CIR model.

Figure 9: Comparison of Method 1 vs. Method 2 for valuation of embedded call option with different numbers of call dates. The input data are given in Table 1.

to maturity. As a result, the value of the callable bond does not change when the number of call dates increases beyond five.

It is worth observing that we find that values of callable bonds computed using Method 1 are higher than those using Method 2. This is illustrated in Table 7 and Figure 9. As noted above, this is to be expected since the issuer has some additional optionality under Method 2. The resulting higher valuation for the call feature is then reflected in a lower valuation for the callable bond.

\section{Conclusions}

In contrast to the claims by Büttler (1995) and Büttler and Waldvogel (1996), we find that a fully numerical PDE method can be used to accurately and efficiently determine the price of callable bonds with notice. The fully numerical PDE method can be used in cases where the analytic 
Green's function cannot be determined, which would normally be the case when time-dependent parameters are used to match the initial term structure. The PDE method converges approximately quadratically, even in the presence of the discontinuity introduced at the call date if Method 2 is used to update the solution. It is, however, important to use an appropriate discretization method for cases where the PDE becomes convection-dominant, both to avoid spurious oscillations, and to ensure that correct boundary conditions are applied. It is also necessary to use the method described in Rannacher (1984) for timestepping, in order to suppress oscillations in delta induced by the discontinuity.

We have also carried out an analysis of the boundary condition required at $r=0$ for the CIR model. As formally specified, the CIR model may require a boundary condition at $r=0$ for certain parameter values. We have proposed a perturbed problem which does not require a boundary condition at $r=0$. We have then utilized a discretization method which ensures convergence of the numerical algorithm for the perturbed problem and which does not require a boundary condition at $r=0$.

\section{Appendix}

In this appendix, we will derive the conditions under which discretization scheme (10) produces a positive coefficient scheme for the PDE (2). In general, a positive coefficient method ensures that spurious oscillations will not be generated. In this appendix, we follow the arguments given in Zvan et al. (2000), which we provide here for the convenience of the reader. For simplicity we will only consider the fully implicit case $(\gamma=1)$; the Crank-Nicolson case $\left(\gamma=\frac{1}{2}\right)$ can be derived analogously (Zvan, Forsyth, and Vetzal, 1998). Substituting $\gamma=1$ into (10) gives

$$
A_{i}\left(\frac{P_{i}^{n+1}-P_{i}^{n}}{\Delta \tau}\right)=\sum_{j \in \eta_{i}} \alpha_{i j}\left(P_{j}^{n+1}-P_{i}^{n+1}\right)+\sum_{j \in \eta_{i}} \overrightarrow{L_{i j}} \cdot \vec{V}_{i} P_{i j+\frac{1}{2}}^{n+1}-r_{i} A_{i} P_{i}^{n+1} .
$$

Letting $\overline{\alpha_{i j}}=\frac{\Delta \tau}{A_{i}} \alpha_{i j}$, equation (36) becomes

$$
P_{i}^{n+1}-P_{i}^{n}=\sum_{j \in \eta_{i}} \overline{\alpha_{i j}}\left(P_{j}^{n+1}-P_{i}^{n+1}\right)+\frac{\Delta \tau}{A_{i}} \sum_{j \in \eta_{i}} \overrightarrow{L_{i j}} \cdot \vec{V}_{i} P_{i j+\frac{1}{2}}^{n+1}-r_{i} \Delta \tau P_{i}^{n+1} .
$$

It follows from the definition of $\overrightarrow{L_{i j}}$ that we can write $(37)$ as

$$
\begin{aligned}
P_{i}^{n+1}-P_{i}^{n}= & \sum_{j \in \eta_{i}} \overline{\alpha_{i j}}\left(P_{j}^{n+1}-P_{i}^{n+1}\right)+ \\
& \frac{\Delta \tau}{A_{i}} \sum_{j \in \eta_{i}} \max \left(\overrightarrow{L_{i j}} \cdot \vec{V}_{i}, 0\right)\left[P_{j}^{n+1}+\frac{\phi\left(q_{j i+\frac{1}{2}}^{n+1}\right)}{2}\left(P_{i}^{n+1}-P_{j}^{n+1}\right)\right]+ \\
& \frac{\Delta \tau}{A_{i}} \sum_{j \in \eta_{i}} \min \left(\overrightarrow{L_{i j}} \cdot \vec{V}_{i}, 0\right)\left[P_{i}^{n+1}+\frac{\phi\left(q_{i j+\frac{1}{2}}^{n+1}\right)}{2}\left(P_{j}^{n+1}-P_{i}^{n+1}\right)\right]-r_{i} \Delta \tau P_{i}^{n+1},
\end{aligned}
$$


where $\phi\left(q_{i j+\frac{1}{2}}^{n+1}\right)$ is the limiter function (see Zvan, Forsyth, and Vetzal, 1998) and

$$
q_{i j+\frac{1}{2}}=\frac{P_{i}^{n+1}-P_{2 u p i j}^{n+1}}{P_{j}^{n+1}-P_{i}^{n+1}} .
$$

Since

$$
\frac{\phi\left(q_{i j+\frac{1}{2}}^{n+1}\right)}{2}\left(P_{j}^{n+1}-P_{i}^{n+1}\right)=\frac{\phi\left(q_{i j+\frac{1}{2}}^{n+1}\right)}{2 q_{i j+\frac{1}{2}}^{n+1}}\left(P_{i}^{n+1}-P_{2 u p i j}^{n+1}\right),
$$

we can rewrite (38) as

$$
\begin{aligned}
P_{i}^{n+1}-P_{i}^{n}= & \sum_{j \in \eta_{i}} \overline{\alpha_{i j}}\left(P_{j}^{n+1}-P_{i}^{n+1}\right)+ \\
& \frac{\Delta \tau}{A_{i}} \sum_{j \in \eta_{i}} \max \left(\overrightarrow{L_{i j}} \cdot \vec{V}_{i}, 0\right)\left[P_{j}^{n+1}+\frac{\phi\left(q_{j i+\frac{1}{2}}^{n+1}\right)}{2}\left(P_{i}^{n+1}-P_{j}^{n+1}\right)\right]+ \\
& \frac{\Delta \tau}{A_{i}} \sum_{j \in \eta_{i}} \min \left(\overrightarrow{L_{i j}} \cdot \vec{V}_{i}, 0\right)\left[P_{i}^{n+1}+\frac{\phi\left(q_{i j+\frac{1}{2}}^{n+1}\right)}{2 q_{i j+\frac{1}{2}}^{n+1}}\left(P_{i}^{n+1}-P_{2 u p i j}^{n+1}\right)\right]-r_{i} \Delta \tau P_{i}^{n+1} .
\end{aligned}
$$

Regrouping the terms of equation (40) gives

$$
\begin{aligned}
&\left(1+\sum_{j \in \eta_{i}} \overline{\alpha_{i j}}-\right.\left.\frac{\Delta \tau}{A_{i}} \sum_{j \in \eta_{i}} \max \left(\overrightarrow{L_{i j}} \cdot \overrightarrow{V_{i}}, 0\right) \frac{\phi\left(q_{j i+\frac{1}{2}}^{n+1}\right)}{2}\right] P_{i}^{n+1}- \\
&\left(\frac{\Delta \tau}{A_{i}} \sum_{j \in \eta_{i}} \min \left(\overrightarrow{L_{i j}} \cdot \vec{V}_{i}, 0\right)\left[1+\frac{\phi\left(q_{i j+\frac{1}{2}}^{n+1}\right)}{2 q_{i j+\frac{1}{2}}^{n+1}}\right]+r_{i} \Delta \tau\right) P_{i}^{n+1}= \\
&\left(\sum_{j \in \eta_{i}} \overline{\alpha_{i j}}+\frac{\Delta \tau}{A_{i}} \sum_{j \in \eta_{i}} \max \left(\overrightarrow{L_{i j}} \cdot \vec{V}_{i}, 0\right)\left[1-\frac{\phi\left(q_{j i+\frac{1}{2}}^{n+1}\right)}{2}\right]\right) P_{j}^{n+1}+ \\
& P_{i}^{n}-\frac{\Delta \tau}{A_{i}} \sum_{j \in \eta_{i}} \min \left(\overrightarrow{L_{i j}} \cdot \vec{V}_{i}, 0\right) \frac{\phi\left(q_{i j+\frac{1}{2}}^{n+1}\right)}{2 q_{i j+\frac{1}{2}}^{n+1}} P_{2 u p i j}^{n+1} .
\end{aligned}
$$


If we require all of the coefficients of $P$ in (41) to be positive, then we must ensure that

$$
\begin{aligned}
1+\sum_{j \in \eta_{i}} \overline{\alpha_{i j}}-\frac{\Delta \tau}{A_{i}} \sum_{j \in \eta_{i}} \max \left(\overrightarrow{L_{i j}} \cdot \overrightarrow{V_{i}}, 0\right) \frac{\phi\left(q_{j i+\frac{1}{2}}^{n+1}\right)}{2}- \\
\qquad \frac{\Delta \tau}{A_{i}} \sum_{j \in \eta_{i}} \min \left(\overrightarrow{L_{i j}} \cdot \overrightarrow{V_{i}}, 0\right)\left[\begin{array}{c}
\phi\left(q_{i j+\frac{1}{2}}^{n+1}\right) \\
2 q_{i j+\frac{1}{2}}^{n+1}
\end{array}\right]+r_{i} \Delta \tau \geq 0
\end{aligned}
$$

and

$$
1-\frac{\phi\left(q_{j i+\frac{1}{2}}^{n+1}\right)}{2} \geq 0 .
$$

Since $\sum_{j \in \eta_{i}} \max \left(\overrightarrow{L_{i j}} \cdot \overrightarrow{V_{i}}, 0\right)+\sum_{j \in \eta_{i}} \min \left(\overrightarrow{L_{i j}} \cdot \overrightarrow{V_{i}}, 0\right)=0$, equation (42) can be written as

$$
\begin{array}{r}
1+r_{i} \Delta \tau+\sum_{j \in \eta_{i}} \overline{\alpha_{i j}}+\frac{\Delta \tau}{A_{i}} \sum_{j \in \eta_{i}} \max \left(\overrightarrow{L_{i j}} \cdot \vec{V}_{i}, 0\right)\left[1-\frac{\phi\left(q_{j i+\frac{1}{2}}^{n+1}\right)}{2}\right]- \\
\frac{\Delta \tau}{A_{i}} \sum_{j \in \eta_{i}} \min \left(\overrightarrow{L_{i j}} \cdot \vec{V}_{i}, 0\right) \frac{\phi\left(q_{i j+\frac{1}{2}}^{n+1}\right)}{2 q_{i j+\frac{1}{2}}^{n+1}} \geq 0 .
\end{array}
$$

Simplifying, condition (43) becomes

$$
1-\frac{\phi\left(q_{j i+\frac{1}{2}}^{n+1}\right)}{2} \geq 0
$$

and

$$
\frac{\phi\left(q_{i j+\frac{1}{2}}^{n+1}\right)}{2 q_{i j+\frac{1}{2}}^{n+1}} \geq 0 .
$$


If conditions (45) and (44) are met, then all coefficients of $P$ in (41) are positive and we can employ the maximum principle. By defining $P_{i}^{\max }=\max \left(P_{j \in \eta_{i}}^{n+1}, P_{i}^{n}\right)$, we can rewrite equation (41) as

$$
\begin{aligned}
& \left(1+r_{i} \Delta \tau+\sum_{j \in \eta_{i}} \overline{\alpha_{i j}}+\frac{\Delta \tau}{A_{i}} \sum_{j \in \eta_{i}} \max \left(\overrightarrow{L_{i j}} \cdot \vec{V}_{i}, 0\right)\left[1-\frac{\phi\left(q_{j i+\frac{1}{2}}^{n+1}\right)}{2}\right]\right) P_{i}^{n+1}- \\
& \left(\frac{\Delta \tau}{A_{i}} \sum_{j \in \eta_{i}} \min \left(\overrightarrow{L_{i j}} \cdot \vec{V}_{i}, 0\right) \frac{\phi\left(q_{i j+\frac{1}{2}}^{n+1}\right)}{2 q_{i j+\frac{1}{2}}^{n+1}}\right) P_{i}^{n+1}= \\
& \left(1+\sum_{j \in \eta_{i}} \overline{\alpha_{i j}}+\frac{\Delta \tau}{A_{i}} \sum_{j \in \eta_{i}} \max \left(\overrightarrow{L_{i j}} \cdot \vec{V}_{i}, 0\right)\left[1-\frac{\phi\left(q_{j i+\frac{1}{2}}^{n+1}\right)}{2}\right]\right)- \\
& \left(\frac{\Delta \tau}{A_{i}} \sum_{j \in \eta_{i}} \min \left(\overrightarrow{L_{i j}} \cdot \vec{V}_{i}, 0\right) \frac{\phi\left(q_{i j+\frac{1}{2}}^{n+1}\right)}{2 q_{i j+\frac{1}{2}}^{n+1}}\right) P_{i}^{\max } .
\end{aligned}
$$

This simplifies to

$$
P_{i}^{n+1} \leq \frac{\lambda}{r_{i} \Delta \tau+\lambda} P_{i}^{\max }
$$

where

$$
\lambda=1+\sum_{j \in \eta_{i}} \overline{\alpha_{i j}}+\frac{\Delta \tau}{A_{i}} \sum_{j \in \eta_{i}} \max \left(\overrightarrow{L_{i j}} \cdot \vec{V}_{i}, 0\right)\left[1-\frac{\phi\left(q_{j i+\frac{1}{2}}^{n+1}\right)}{2}\right]-\frac{\Delta \tau}{A_{i}} \sum_{j \in \eta_{i}} \min \left(\overrightarrow{L_{i j}} \cdot \overrightarrow{V_{i}}, 0\right) \frac{\phi\left(q_{i j+\frac{1}{2}}^{n+1}\right)}{2 q_{i j+\frac{1}{2}}^{n+1}} .
$$

If conditions (45) and (44) are met then $\lambda>0$ and (47) can be written as

$$
P_{i}^{n+1} \leq \frac{1}{1+\frac{r_{i} \Delta \tau}{\lambda}} P_{i}^{\max } \leq P_{i}^{\max }
$$

Similarly, if $P_{i}^{\min }=\min \left(P_{j \in \eta_{i}}^{n+1}, P_{i}^{n}\right)$ then (noticing that $\lambda \geq 1$ )

$$
P_{i}^{\min } \frac{1}{1+r_{i} \Delta \tau} \leq P_{i}^{n+1}
$$

Consequently,

$$
P_{i}^{\min } \frac{1}{1+r_{i} \Delta \tau} \leq P_{i}^{n+1} \leq P_{i}^{\max } .
$$




\section{References}

Bank for International Settlements (2000). The Global OTC Derivatives Market at End-December 1999. Press release dated May 18, available from www.bis.org.

Beaglehole, D. R. and M. S. Tenney (1991). General solutions of some interest rate-contingent claim pricing equations. Journal of Fixed Income 2, 69-83.

Bhar, R., C. Chiarella, N. El-Hassan, and X. Zheng (2000). The reduction of forward rate dependent volatility HJM models to Markovian form: Pricing European bond options. Journal of Computational Finance 3 (Spring), 47-72.

Bliss, R. R. and E. I. Ronn (1998). Callable U.S. Treasury bonds: Optimal calls, anamolies, and implied volatilities. Journal of Business 71, 211-252.

Brennan, M. J. and E. S. Schwartz (1977). Savings bonds, retractable bonds and callable bonds. Journal of Financial Economics 5, 67-88.

Büttler, H.-J. (1995). Evaluation of callable bonds: Finite difference methods, stability and accuracy. Economic Journal 105, 374-384.

Büttler, H.-J. and J. Waldvogel (1996). Pricing callable bonds by means of Green's function. Mathematical Finance 6, 53-88.

Cox, J. C., J. E. Ingersoll, Jr., and S. A. Ross (1985). A theory of the term structure of interest rates. Econometrica 53, 385-407.

Crabbe, L. E. and P. Nikoulis (1997). The putable bond market: Structure, historical experience, and strategies. Journal of Fixed Income 7 (December), 47-60.

Davenport, J., Y. Siret, and E. Tournier (1989). Computer Algebra (Second ed.). Academic Press, San Diego, CA.

Duffie, D. and R. Kan (1996). A yield factor model of interest rates. Mathematical Finance 6, $379-406$.

Duffie, D. and K. J. Singleton (1999). Modeling term structures of defaultable bonds. Review of Financial Studies 12, 687-720.

Feller, W. (1951). Two singular diffusion problems. Annals of Mathematics 54, 173-182.

Heath, D., R. Jarrow, and A. Morton (1992). Bond pricing and the term structure of interest rates: A new methodology for contingent claims valuation. Econometrica 60, 77-105.

Houston, P., C. Schwab, and E. Süli (2000). Discontinuous hp-finite element methods for advectiondiffusion problems. Working paper, Oxford University Computing Laboratory.

Hughston, L. (Ed.) (1996). Vasicek and Beyond: Approaches to Building and Applying Interest Rate Models. Risk Publications, London.

Hull, J. C. (2000). Option, Futures, $\&$ Other Derivatives (Fourth ed.). Prentice Hall, Inc., Upper Saddle River, N.J. 
Hull, J. C. and A. White (1990). Pricing interest rate derivative securities. Review of Financial Studies 3, 573-592.

Jamshidian, F. (1987). Pricing of contingent claims in the one-factor term structure model. Working paper, Merrill Lynch Capital Markets, reprinted as Chapter 7 of Hughston (1996).

Johnson, C. (1987). Numerical Solutions of Partial Differential Equations By the Finite Element Method. Cambridge University Press, Cambridge.

Kröner, D. (1997). Numerical Schemes for Conservation Laws. Chichester, New York.

Longstaff, F. A. and B. A. Tuckman (1994). Calling nonconvertible debt and the problem of related wealth transfer effects. Financial Management 23 (Winter), 21-27.

Mauer, D. C. (1993). Optimal bond call policies under transactions costs. Journal of Financial Research 16, 23-37.

Oleinik, O. A. and E. V. Radkevic (1973). Second Order Equations with Nonnegative Characteristic Form. American Mathematical Society, Providence, R.I.

Rannacher, R. (1984). Finite element solution of diffusion problems with irregular data. Numerische Mathematik 43, 309-327.

Ritchken, P. and L. Sankarasubramanian (1995). Volatility structure of forward rates and the dynamics of the term structure. Mathematical Finance 5, 55-72.

Ritger, P. D. and N. J. Rose (1968). Differential Equations with Applications. McGraw-Hill, NewYork.

Vasicek, O. (1977). An equilibrium characterization of the term structure. Journal of Financial Economics 5, 177-188.

Zvan, R., P. A. Forsyth, and K. R. Vetzal (1998). Robust numerical methods for PDE models of Asian options. Journal of Computational Finance 1 (Winter), 39-78.

Zvan, R., P. A. Forsyth, and K. R. Vetzal (2000). A finite volume approach for contingent claims valuation. Working paper, University of Waterloo, Department of Computer Science.

Zvan, R., K. R. Vetzal, and P. A. Forsyth (1998). Swing low, swing high. Risk 11 (March), 71-75. 